\title{
Lapurdum
}

Euskal ikerketen aldizkaria | Revue d'études basques |

Revista de estudios vascos | Basque studies review

$11 \mid 2006$

Numéro XI

\section{Distribution des patronymes et structure des populations dans les Pyrénées occidentales}

\section{Pierre Darlu et Bernard Oyharçabal}

\section{OpenEdition \\ Journals}

Édition électronique

URL : http://journals.openedition.org/lapurdum/266

DOI : 10.4000/lapurdum.266

ISSN : 1965-0655

\section{Éditeur}

IKER

\section{Édition imprimée}

Date de publication : 1 novembre 2006

Pagination : 137-165

ISBN : 978-2-86781-474-7

ISSN : $1273-3830$

\section{Référence électronique}

Pierre Darlu et Bernard Oyharçabal, «Distribution des patronymes et structure des populations dans les Pyrénées occidentales », Lapurdum [En ligne], 11 | 2006, mis en ligne le 01 avril 2008, consulté le 20 avril 2019. URL : http://journals.openedition.org/lapurdum/266 ; DOI : 10.4000/lapurdum.266 


\section{Distribution des}

\section{patronymes et structure des populations dans les Pyrénées occidentales}

\author{
Pierre DARLU \\ INSERM U535 \\ Génétique épidémiologique et \\ Structure des populations humaines \\ Hôpital Paul Brousse \\ BP 1000 \\ 94817 VILLEJUIF CEDEX \\ darlu@vjf.inserm.fr
}

\author{
Beñat OYHARÇABAL \\ UMR5478, \\ Centre de recherches \\ sur la langue basque et \\ l'expression en langue basque \\ 28 , rue Lormand \\ 64100 BAYONNE \\ b.oyharcabal@wanadoo.fr
}

\section{Introduction}

Les recherches sur les noms de familles connaissent, depuis quelques années, des développements considérables consécutifs à l'informatisation des sources et aux raffinements méthodologiques avec lesquels ces sources sont exploitées. Les registres paroissiaux, les actes de naissances, mariages ou décès sont en passe d'être systématiquement dépouillées et informatisés sur de larges zones géographiques et sur plusieurs dizaines de générations, ouvrant ainsi de vastes champs d'investigation. L'engouement pour l'onomastique tient au fait qu'elle permet à chacun de remonter en profondeur les généalogies de ses proches parents, et de les insérer dans un contexte géographique bien circonscrit, le nom gardant bien souvent l'empreinte de ses origines géographiques. Cependant, il serait particulièrement dommage de s'arrêter à des approches qui s'intéressent plus à décrire des histoires individuelles qu'à fournir des informations à valeur plus générale. Pour accéder à de telles informations, il devient nécessaire d'invoquer l'outil statistique qui permet de faire émerger, de la profusion des noms ou des histoires individuelles, des considérations sur l'organisation spatiale des populations, sur la réalité des frontières géographique, historique, culturelle ou linguistique qui les traversent, mais aussi sur la mobilité et les échanges migratoires survenus dans le passé de part et d'autre de ces frontières 
L'ambition de cette note est de proposer quelques réponses à ces questions, dans le contexte du Pays Basque de France, du Béarn et de la Bigorre et en s'appuyant sur des méthodologies récentes.

De nombreuses études onomastiques ont déjà été publiées sur ces régions, et l'on ne saurait être ici exhaustif. Lorsqu'elle ne cumulent pas les deux types d'objets dans des monographies (Irigaray 1955, Goyheneche 1966, Iglesias 2000, Elosegi 2005), elles portent en majorité sur les toponymes (voir notamment Orpustan 1991, Grosclaude 1991, Grosclaude \& Le Nail 2000, Boyrie-Fénié 2005), plus rarement sur les domonymes (en particulier, Orpustan 2000a), lesquels, il est vrai, sont souvent à l'origine de patronymes, notamment en Pays Basque. Les travaux sur les patronymes, bien que moins nombreux et de qualité plus inégale, existent également. Il peut s'agir de recensements sous forme de dictionnaires locaux tels que ceux réalisés par Larronde (1986) en Bigorre, et Pées (2003) à Bayonne, ou plus généraux comme ceux d'Oyhamburu (1991) ou Larronde (1997). Il convient enfin de mentionner spécialement les travaux réalisés par des philologues ou diachroniciens spécialistes (Michelena 1953, Grosclaude 1992), auxquels on joindra aussi les études d'anthroponymie (étendue éventuellement à la théonymie pour le début de l'ère) portant sur des périodes plus ou moins éloignées (Berganton 1977, Luchaire 1881, Gorrochategi 1984, Orpustan 2000b). Certaines approches insistent sur le rôle spécifique joué par le nom de famille dans ces régions (Jakobi et al., 1976) tandis que d'autres se placent davantage sur un plan statistique ou démographique pour souligner la présence de discontinuités spatiales entre le Pays Basque, le Béarn et la Bigorre, et mesurer les flux migratoires entre ces trois régions (Darlu et al. 2001) ou préciser les origines de l'expansion démographique de villes comme Tarbes (Degioanni et Darlu, 2001).

\section{Les données}

Les données patronymiques qui sont étudiées dans cet article sont issues du fichier patronymique de l'INSEE (1985). Ce fichier fournit le nombre des naissances enregistrées sous chaque patronyme par commune. Il inclut toutes les personnes nées entre 1891 et 1940 et non décédées en 1972. On peut donc considérer qu'il s'agit de données représentatives de la richesse relative en différents patronymes. Deux biais pourraient être évoqués. Le premier concerne l'ampleur de la période considérée, correspondant à près de 3 générations. Pendant une telle durée, la composition patronymique a du se modifier, en particulier par l'apport de noms de migrants. La prise en compte de tels noms « migrants » est d'ailleurs une stratégie commode pour inférer des taux d'immigration (Darlu et al. 1996, Degioanni et Darlu, 2001). Cependant, ces noms fraîchement arrivés sont en fréquences nécessairement rares et n'auront donc que peu de poids dans les analyses présentées ici qui portent essentiellement sur les noms les plus fréquents. Le deuxième biais concerne le fait que seules les personnes encore vivantes en 1972 ont été informatisées par l'INSEE. Mais, sauf à considérer qu'il s'est produit une mortalité différentielle selon le nom de famille, par exemple à l'occasion de la Première Guerre Mondiale, on peut négliger ce biais à l'échelle de ce travail. Enfin, 
les noms ne sont portés qu'en lettres capitales, sans accent, ce qui peut introduire occasionnellement quelques possibles confusions. Nous en verrons un exemple.

Un autre problème est celui de l'homogénéisation orthographique ou lemmatisation des noms. En effet, le fichier INSEE, comme d'ailleurs la plupart des fichiers nominatifs, comporte des variations orthographiques autour d'un même nom, sans que l'on puisse véritablement trancher entre une variation signifiante et une erreur de transcription dont l'origine peut aussi bien se situer dans le registre princeps (actes d'état-civil) que résulter d'une saisie informatique erronée. Cependant, il s'avère, fort heureusement, que ces noms "dérivés " ont une très faible fréquence et restent rares. Ils ne peuvent donc modifier de manière conséquente les analyses statistiques qui se fondent essentiellement sur les hautes fréquences patronymiques. En revanche, lorsque deux ou trois formes orthographiques d'un même nom se retrouvent en fréquences proches, il est délicat de vouloir les regrouper, dans la mesure où ces variations peuvent répondre à des distributions géographiques différentes. Dans ce cas nous avons décidé de les conserver pour ne pas perdre l'éventuelle information géographique qu'ils contiennent. Ce point sera illustré lors de la discussion.

Comme le nombre de naissances par commune peut s'avérer très faible, particulièrement dans une région où les petites communes ne sont pas rares, l'unité géographique qui nous a semblé la plus pertinente est celle du canton. Toutes les naissances, et les noms qui les accompagnent, relevant des communes d'un même canton, ont donc été cumulées, en se restreignant aux cantons des départements des Pyrénées-Atlantiques et des Hautes-Pyrénées (tableau 1, figure 1). En revanche, les cantons correspondant à des agglomérations trop considérables ou qui ont été des pôles trop évident d'attraction pour des immigrants venus des régions avoisinantes, plus lointaines ou même de l'étranger, n'ont pas été prises en considération pour toutes les analyses. Il s'agit des agglomérations de BayonneBiarritz-Anglet, Pau-Billère-Idron, Tarbes-Aureilhan-Séméac-Laloubère. Ce travail se focalise donc sur 60 cantons (tableau 1 p. 146) à dominante rurale.

\section{Méthodes}

\section{Diversité patronymique et indice d'isonymie}

La diversité patronymique en un lieu donné $i$ peut être quantifiée par le rapport entre le nombre de patronymes différents en ce lieu, $S_{i}$, et le nombre $N_{i}$ de naissances recensées. Exprimé en pourcentage, ce rapport représente donc le nombre de patronymes différents enregistrés pour 100 naissances. C'est ce que l'on peut appeler le « potentiel patronymique » ou l'indice de condensation (Chareille, 1992).

$$
P_{i}=100 \times \frac{S_{i}}{N_{i}}
$$


L'inverse de ce rapport donne le nombre de personnes naissant, en moyenne, sous le même patronyme.

Une autre façon d'exprimer la diversité patronymique consiste à calculer la probabilité de tirer au hasard deux patronymes identiques dans la même localité $i$. Cette probabilité, ou indice d'isonymie $l i$, est d'autant plus élevée que la diversité patronymique est faible.

$$
I_{i}=\sum_{k} f_{i k}^{2}
$$

où $f_{i k}$ est la fréquence du kième patronyme dans la localité $i$ et où la somme est effectuée sur l'ensemble des patronymes $k$.

Les statistiques sur cet indice de diversité sont données dans le tableau 2 et sa distribution géographique par canton dans la figure 2 .

\section{Distance patronymique}

L'objectif est de comparer des listes de noms relevées dans plusieurs régions géographiques et de déterminer un degré de ressemblance ou de différence entre ces listes, prenant en compte les fréquences de ces noms dans les différentes régions. L'idée est que deux régions en contact et ayant établi des échanges réguliers sur plusieurs générations auront plus de chance de présenter des stocks patronymiques voisins que deux régions séparées par des barrières géographiques, culturelles ou administratives. L'un des indices de ressemblance le plus utilisé est dérivé de la génétique des populations (Nei, 1973)

$$
\varphi_{\mathrm{ij}}=\left[\frac{\sum_{\mathrm{k}}\left(\mathrm{f}_{\mathrm{ik}} \mathrm{f}_{\mathrm{jk}}\right)}{\left(\sum_{\mathrm{k}} \mathrm{f}_{\mathrm{ik}}^{2} \sum_{\mathrm{k}} \mathrm{f}_{\mathrm{jk}}{ }^{2}\right)^{\frac{l}{2}}}\right]
$$

où $f_{i k}$ et $f_{j k}$ sont les fréquences du patronyme $k$ dans les régions $i$ et $j$ respectivement. Le numérateur est dérivé du coefficient de parenté entre deux populations défini par Malécot (1966) et standardisé au dénominateur pour tenir compte de la diversité patronymique des deux régions $i$ et $j$ telle qu'elle est définie plus haut. Cet indice de ressemblance est souvent transformé en indice de distance de la façon suivante :

$$
\delta_{i j}=-\ln \left(\varphi_{i j}\right)
$$

Cette distance est maximale, lorsque aucun patronyme de la région $i$ ne se retrouve dans la région $j$. Elle est d'autant plus proche de 0 que la distribution des patronymes dans la région $i$ est comparable à celle de la région $j$. En se basant sur 
la matrice des distances patronymiques entre toutes les régions prises deux à deux, il est possible d'établir les relations entre les corpus de noms de plusieurs régions simultanément.

L'exploitation de cette matrice de distances peut se faire selon plusieurs orientations. Nous en proposons deux dans cette note, une méthode d'agglomération dite du Neighbor-joining ou NJ (Saitou et Nei, 1987 ; Degioanni et al., 2001) et une méthode de recherche de barrières (Manni et al., 2004). Les résultats sont testés par des méthodes statistiques de rééchantillonnage (bootstrap) (Felsenstein, 1985, 2004).

\section{L'arbre de NJ}

Il s'agit d'une méthode qui transforme la matrice des distances patronymiques entre cantons en un arbre dichotomique, tout en minimisant la perte d'information. Dans cet arbre, les cantons les plus proches, en terme de distance patronymique, sont groupés ensemble par un algorithme d'agglomérations successives. Les branches séparant des groupes de cantons sont plus ou moins longues selon leur degré de divergence patronymique. Une longue branche est donc le signe d'une différentiation patronymique importante. Par ailleurs, l'application du bootstrap permet d'attribuer à ces regroupements une certaine confiance ou robustesse. La méthode consiste à recalculer 100 fois une matrice de distance basée sur les patronymes, mais en attribuant au hasard à chacun d'eux un certain poids $(0,1,2,3$ ou plus), équivalent à un tirage aléatoire des patronymes avec remise. Puis, de ces 100 matrices, 100 arbres sont reconstruits par la méthode NJ. Par suite des tirages aléatoires, ces arbres peuvent, évidemment, présenter des discordances dans leur structure. Pour mesurer ces discordances, on attribue un pourcentage à chacun des regroupements entre cantons. Ce pourcentage correspond donc au nombre de fois, sur les 100 répétitions effectuées, où un même regroupement de cantons à été obtenu. Sur la figure 3, les regroupements retrouvés dans plus de $90 \%$ des tirages aléatoires sont représentés en traits épais. Ils définissent donc des structures «fortes » peu dépendantes de l'échantillonnage de patronymes.

\section{Les Barrières}

Cette méthode (Monmonier, 1973) consiste à rechercher, dans la matrice de distances patronymiques, les distances entre cantons adjacents les plus élevées. Ce sont elles qui permettent de définir des «barrières » entre cantons. L'algorithme commence par chercher les deux cantons adjacents présentant entre eux la distance patronymique la plus grande. C'est l'amorce d'une première "barrière ». Puis partant de l'un des deux cantons, la barrière est prolongée vers le canton adjacent qui présente la distance la plus grande immédiatement inférieure. De proche en proche, la procédure conduit à prolonger ainsi la délimitation de la barrière, jusqu'à "sortir » de la carte ou de «boucler » sur un canton, définissant ainsi une première barrière. La procédure est relancée ensuite, à la recherche d'une 
deuxième barrière, en partant cette fois de la distance entre cantons adjacents immédiatement inférieure par rapport à celle retenue pour la première barrière. L'algorithme est stoppé lorsque le nombre de frontière est jugé pertinent. De plus, une procédure de bootstrap permet d'attribuer une certaine confiance à chacune de ces barrières. De la même façon que l'on a définit des groupes de cantons ayant une proportion de bootstrap de $90 \%$ dans l'arbre construit par $N J$, de même peuton définir des barrières à 99\%, 90\%, 80\% etc.... La figure 4 illustre la méthode, en distinguant les barrières par des traits plus ou moins épais en fonction des valeurs de bootstrap obtenues. Le logiciel BARRIER (Manni et al., 2004) a été utilisé pour ces analyses.

\section{Résultats et discussion}

Une première réflexion porte sur la pertinence de notre refus de lemmatiser les noms. Les raisons invoquées tiennent à la fois au fait que les formes rares ne perturbent pas les analyses statistiques et que les formes fréquentes peuvent révéler des disparités géographiques qu'il ne faut pas "noyer » par une lemmatisation abusive dans la mesure où elles peuvent résulter de variations dialectales, ou même simplement de traditions orthographiques divergentes (Etcheverry vs Etcheberry), parfois influencées par des procédés analogiques (généralisation de l'emploi de la préposion d- dans certains patronymes basques à initiale vocalique). Nous en donnons ici quelques exemples (voir annexe 2 p. 161165). Ainsi, observe-t-on un glissement progressif, d'ouest en est, des noms DUHAU, DUFAU et DUFFAU. La présence de DUHAU en Pays Basque se situe essentiellement dans les cantons de La-Bastide-Clairence, Saint-Palais, Saint-Pierre d'Irube, confirmant donc son origine occitane. L'orthographe DUFFAU, en revanche, se retrouve presque exclusivement autour du Lannemezan et de Maubourguet. Selon un même mouvement d'ouest en est, DAGUERRE, fréquent à Ustaritz, se transforme en AGUERRE plus à l'est à Saint-Palais et Iholdy, puis en AGUER à Mauléon-Licharre. Autre exemple, celui d'HIRIGOYEN, qui a son centre de gravité à Hasparren, tandis que celui d'IRIGOYEN se situe plus au sud, à Tardets et Saint-Jean-Pied-de-Port. Citons également le cas de HOURCADE, très béarnais, qui présente une fréquence élevée à Saint-Palais, tandis que l'orthographe FOURCADE se retrouve presque exclusivement en Bigorre. Enfin l'orthographe "CASAUX » domine au cour du Béarn, jusqu'en bordure nord du Pays Basque, tandis que l'orthographe CAZAUX » domine au cœur de la Bigorre. Tous ces exemples montrent bien qu'il ne serait simplement pas raisonnable de sommer les statistiques concernant des noms aux origines étymologiques identiques dans la mesure où ils présentent des distributions géographiques aussi contrastées.

Le tableau 2 et la figure 2 (p. 149) soulignent les importantes disparités régionales en ce qui concerne la diversité patronymique. La Bigorre est la région où les cantons sont les moins pourvus en patronymes. On y trouve près de deux fois moins de patronymes qu'en Pays basque. L'indice de condensation $P$ y est plus élevé qu'en Béarn ou qu'en Pays basque. Ce sont les raisons pour lesquelles la probabilité de trouver deux personnes portant le même nom se trouve être bien 
plus élevée en Bigorre qu'en Béarn ou qu'au Pays Basque. C'est ce que mesure l'indice d'isonymie. Sur les 24 cantons de Bigorre, l'isonymie est évaluée à $0.65 .10^{-3}$ en moyenne, alors qu'elle est de $0.35 .10^{-3}$ en Pays basque et $0.30 .10^{-3}$ en Béarn. La distribution cantonale de l'isonymie fait l'objet de la figure $2 \mathrm{~b}$. Par ailleurs, le pourcentage d'hapax, c'est-à-dire le pourcentage de noms portés par une seule personne, est bien supérieur dans les cantons de Bigorre (43.5\%) par rapport à ce qu'il est en Pays Basque (40.5\%) et en Béarn (37.0\%). Notons cependant que ces noms « uniques » n'interviennent que de façon très marginales dans l'estimation de la diversité patronymique telle qu'elle est estimée dans la formule de l'isonymie, leur fréquence $f_{i k}$ étant très faible.

Si la diversité patronymique semble plus faible en Bigorre qu'en Béarn et qu'en Pays Basque, d'importants contrastes s'observent néanmoins à l'intérieur de chacune de ces régions (voir la figure 2a et les écart-types du tableau 2). En Bigorre, les hautes vallées (Aure, Campan, Aucun) et le pourtour du plateau de Lannemezan présentent une diversité patronymique plus faible, soulignant leur isolement relatif, que celle observée dans la Bigorre de l'ouest, enrichie par la proximité du Béarn. De même, en Pays Basque, un certain contraste s'observe entre la Basse-Navarre d'une part et la moitié nord de la Soule et le Labourd d'autre part, cette dernière région montrant une plus grande diversité patronymique, certainement liée à la proximité des grandes agglomérations et de la côte, pourvoyeuses de patronymes exogènes.

Enfin, une autre façon d'apprécier la diversité consiste à se pencher sur les noms les plus fréquents dans chacune des régions. En Bigorre, les 100 noms les plus fréquents représentent 26,7\% des naissances, mais seulement $22.8 \%$ en Béarn et $20.5 \%$ en Pays Basque. Pour les 200 noms les plus fréquents, ces proportions sont, respectivement, $37.8 \%, 31.5 \%$ et $28.8 \%$. Il y a donc une concentration des noms nettement plus importante en Bigorre.

L'examen de la liste des 100 et 200 noms les plus fréquents (annexe 1) dans chacune des trois régions apporte également des informations non seulement sur leur fréquences respectives, mais également sur l'ampleur de leur diffusion à l'intérieur d'une région et entre régions. Il s'avère que très peu de noms de cette liste se retrouvent avec une fréquence supérieure à $0.1 \%$, simultanément dans les trois régions. C'est seulement le cas des CLAVERIE, LABAT, LABORDE et VERGEZ. Si l'on abaisse le seuil à $0.5 \%, 23$ patronymes supplémentaires répondent à ce critère de présence simultanée dans les trois régions (ABADIE, BARTHE, BORDENAVE, BORDES, CAPDEVILLE, CARRERE, CAZAUX, CAZENAVE, FOURCADE, GARCIA, LACOSTE, LAFFITTE, LAMARQUE, LAPORTE, LARRE, LARROQUE, LARTIGUE, LASSALLE, LASSERRE, LAVIGNE, PEREZ, PUCHEU, SOULE).

Dans cette liste, le nom LARRE mérite une observation. En effet, sous une telle graphie, c'est le seul patronyme de cette liste qui soit d'origine basque, et cette extension géographique surprend, d'autant que le nom se trouve notamment en Bigorre (cantons de Tournay, Pouyastruc, et Trie-sur-Baïse), près de Lannemezan, avec sa plus forte présence dans les zones partiellement ou totalement occitanophones du Pays Basque et de ses marges (cantons de Labastide-Clairence et Saint-Pierre-d'Irube). Mais ceci est sans doute le résultat d'une coïncidence de graphie de deux patronymes distincts : l'un basque, l'autre occitan. Ce dernier connait plusieurs graphies dont l'une, bien attestée dans l'annuaire téléphonique, correspond à celle de LARRE, mais avec un accent aigu sur le -e final, signe qui est 
perdu dans la base de données informatisée que nous utilisons. D'où la coïncidence et la confusion possible. Observons que si le patronyme LARRE est bisyllabique en basque, avec un -e final prononcé semi fermé, cet -e final n'est jamais accentué dans les graphies du patronyme basque romanisé; il est d'ailleurs muet dans sa prononciation non basque usuelle souvent suivie dans l'emploi du patronyme par les bascophones de France eux-mêmes.

Parmi les 100 noms les plus fréquents, seulement 43 noms se retrouvent simultanément en Pays Basque et en Béarn tout en étant absent en Bigorre. Trois quarts d'entre eux sont d'origine basque. En revanche, 25 noms se trouvent en Béarn et Bigorre tout en étant absent en Pays Basque, et parmi eux, aucun nom basque, et seulement 9 noms se trouvent en Pays basque et Bigorre mais sont absents en Béarn (BAZERQUE, DUTHU, ECHEVERRIA, ELISSALDE, ETCHETO, EYHERABIDE, HEGUY, LARRONDE, TOUJAS).

Ainsi, l'inégale distribution de noms basques en Béarn ou Bigorre et par rapport à celle des noms Béarnais ou Bigourdans en Pays Basque reflète une certaine directionnalité dans les mouvements de populations, avec une migration préférentielle des cantons de l'est vers le Pays Basque plutôt que l'inverse, comme il a déjà été signalé ailleurs (Darlu et al., 2001).

Les descriptions précédentes permettent de comprendre comment la prise en compte des gradients de fréquence de l'ensemble des patronymes conduit à cerner les affinités patronymiques entre cantons et à délimiter les zones entre lesquelles se placent des transitions patronymiques significatives. De fait, la représentation arborée de la figure 3 et les positions des barrières dans l'espace des cantons (figure 4) soulignent bien l'individualité des trois régions. Les cantons du Pays Basque se regroupent ensemble, de façon bien séparée des cantons du Béarn, par une relativement longue branche, donc une grande distance patronymique, et une valeur de bootstrap élevée ( $\mathrm{BP}=100 \%)$. De même, la Bigorre se distingue du Béarn par deux branches, l'une vers Maubourguet et Castelnau-Rivierre-Basse $(\mathrm{BP}=100 \%)$, l'autre rassemblant tous les autres cantons $(\mathrm{BP}=93 \%)$. En revanche, le Béarn s'avère bien moins structuré, si l'on excepte sa partie ouest, avec Lasseube, Monein, Arudy, Oloron, Accous et Aramits. Le reste se situe dans une zone intermédiaire où les échanges entre cantons ont du créer, au fil des générations, une homogénéisation des noms de famille, de place en place.

A l'intérieur de la Bigorre, la structuration reste forte, avec des individualisations nettes des cantons isolés dans des vallées, comme ceux de la haute et basse Vallée d'Aure, ceux de la Vallée de Campan ou la vallée d'Argelès-Gazost. Les cantons de piémont, pour les mêmes raisons que les cantons du Béarn, restent également peu différenciés.

Du coté du Pays Basque, la structuration est encore plus évidente, comme le confirme la figure $5 \mathrm{a}$ et $5 \mathrm{~b}$. Cette figure souligne l'existence de quatre groupes de cantons, chacun d'eux fortement défini par des valeurs de bootstrap élevées. Si l'on met à part la zone incluant Bidache, La Bastide et Saint-Pierre-d'Irube, occitanophone ou sous influence, la structuration s'effectue selon les trois zones traditionnelles, le Labourd, la Basse Navarre et la Soule. La zone côtière et le sud du Labourd constituent des entités à peine différentiées des précédentes. Notons une apparente contradiction entre la figure 3 et la figure 5 à propos du Pays Basque. Dans la figure 5, seuls les cantons basques, et les noms qui s'y trouvent, ont été pris 
en compte, neutralisant ainsi les attractions créées par les cantons béarnais et bigourdans qui influencent la structure de la figure 3. Dans la figure 5, toutes les valeurs de boostrap définissant les groupes sont supérieures à 93\% à l'exception du groupe formé de Saint-Jean-Pied-de-Port et Saint-Étienne de Baigorry (88\%), Les représentations en nuances de couleurs de la figure 5 montrent la présence de ces quatre groupes, représentés par quatre couleurs dont l'intensité, du foncé vers le clair décrit leur emboîtement comme l'indique l'arbre reconstruit par NJ. Cet arbre a le mérite de faire figurer les longueurs de branches, indiquant les grandes différentiations de certains cantons comme Hendaye, ville frontière, Bidache proche de la Chalosse, ou Mauléon et Tardets, cantons basques souletins (donc dialectalement distincts) et jouxtant le Béarn.

\section{Conclusions}

Les patronymes nous ont fourni un outil efficace pour décrire la structuration des populations. L'information est à la fois abondante et précise et permet donc des inférences solides. L'analyse de la diversité permet de rendre compte du degré d'isolation des populations et les affinités patronymiques entre populations permettent d'inférer l'intensité des échanges qui ont pu se produire entre elles tout $\mathrm{au}$ long des générations. Les représentations proposées dans ce travail ne recouvrent qu'une période de 50 ans, de la fin du XIX ${ }^{\mathrm{e}}$ siècle au début du XX $\mathrm{XX}^{\mathrm{e}}$, période pendant laquelle la stabilité patronymique est avérée (Darlu et al., 2001). Elles décrivent une réalité complexe où le cloisonnement entre régions parait particulièrement fort, mais où le compartimentage se poursuit également jusqu'à l'intérieur même de ces régions. Il serait intéressant d'étudier la distribution des patronymes sur des périodes antérieures afin de préciser la stabilité ou l'évolution de ces structures au cours des générations, car il n'est pas évident que la structure actuelle recoupe celle en vigueur au $\mathrm{XVIII}^{\mathrm{e}}$ siècle par exemple. De plus, l'observation des patronymes sur plusieurs longues périodes permet d'aller plus loin et d'estimer les flux migratoires entre régions (Darlu et al., 2001), de préciser l'origine des migrants (Degioanni et Darlu, 2001) et donc de mieux comprendre le renouvellement des populations. Cela n'est pas sans implications génétiques, dans la mesure où le nom de famille se transmet de père en fils. Les conclusions tirées de l'étude des patronymes sont donc a priori transposables en terme de génétique des populations. Les récents programmes de recherche génétique entrepris dans ce domaine se trouvent confortés dans leur stratégie d'investigation par cette étude des patronymes et, en retour, permettront d'en vérifier la réalité biologique. Enfin, les données traitées dans ce travail ne font aucune distinction étymologique ou linguistique entre les patronymes. Sans doute pourrait-on aller plus loin, en distinguant les patronymes en fonction de leur origine linguistique. Cette distinction permettrait d'apprécier quantitativement la perméabilité des région linguistiquement basques à l'influence béarnaise et réciproquement. Ce travail reste à faire. 


\section{Bibliografia}

- Berganton, M.-F. 1977. Le dérivé du nom individuel au moyen Age en Béarn et en Bigorre : usage officiel, suffixes et formation. Éditions du CNRS, Paris.

- Boyrie-Fénié, B. 2005. Dictionnaire toponymique. Landes et Bas-Adour. Editions Cairn, Pau.

- Chareille, P. 1992. Éléments pour un traitement statistique des données anthroponymiques. In. : Genèse médiévale de l'Anthroponymie moderne, Tome II-2. Bourin M. et Chareille P. Eds. Publication de l'université de Tours, Tours.

- Darlu, P., A. Degioanni, et al. (2001). Les cloisonnements dans les Pyrénées occidentales. Evolution, du $\mathrm{XIX}^{\mathrm{e}}$ siècle à nos jours. Le Patronyme. Histoire, anthropologie, société. G. Brunet, P. Darlu and G. Zei. Paris, CNRS-Editions: 173-187.

- Darlu, P., A. Degioanni, et al. (1996). Patronymes et migrations dans les populations humaines. Analyse spatiale de données biodémographiques. BocquetAppel. J.P. and D. Courgeau. Paris, John Libbey/INED: 225-253.

- Degioanni, A. and P. Darlu (2001). "A Bayesian approach to infer geographical origins of migrants through surnames." Annals of Human biology 28(5): 537-545.

- Elosegi, X. 2005. Sara. Etxeak eta deiturak lau mendez (XVI-XIX). Eusko Ikaskuntza. San Sebastian - Donostia.

— Felsenstein, J. 1985. Confidence Limits on Phylogenies : An Approach Using the Bootstrap. Évolution (39): 783-791.

- Felsenstein, J. 2004. PHYLIP (Phylogeny Inference Package) version 3.6b. Distributed by the author. Department of Genome Sciences, University of Washington, Seattle.

- Gorrochategui Churruca, J. 1984. Estudio sobre la onomástica indígena de Aquitanía. Universitad del Pais Vasco - Euskal Herriko Unibertsitatea, Bilbao.

- Goyheneche, E. 1966. Onomastique du Nord du Pays Basque (XI $I^{e}-X V^{e}$ siècles). Ouvrage non publié. Manuscrit du Musée Basque de Bayonne.

- Grosclaude, M. 1991. Dictionnaire toponymique des communes du Béarn. Seconde édition, 2006. Editions Cairn, Pau \& Reclams, Serras-Morlas.

- Grosclaude, M. 1992. Dictionnaire étymologique des noms de famille gascons. Seconde édition, 2003. Editions Per Noste \& Radio Pais, Pau.

— Grosclaude, M. \& Le Nail, J.F. 2000. Dictionnaire toponymique des communes des Hautes-Pyrénées. Conseil Général des hautes-Pyrénées, Tarbes.

— Iglesias, H. 2000. Noms de lieux et de personnes à Bayonne, Anglet et Biarritz au XVIII siècle. Elkarlanean, Bayonne.

— INSEE (1985). Registre français des noms patronymiques.

— Irigaray, A. 1955. "Antroponimos medievales de Navarra". Principe de Viana XVI, Pamplona.

- Jakobi, L., P. Fernet, Jacquard A. (1976). "Transmission des noms et reconstruction des généalogies. Influence de coutumes en Béarn." Biométrie Humaine XI: 69-79. 
— Larronde, C. (1986). Les noms de famille à Vic-Bigorre du XIII ${ }^{e}$ au XX $X^{e}$ siècle. Les Amis du vieux Pics. Vic-en-Bigorre.

— Larronde, C. (1997). Les noms de famille gascons, Société Académique des HautesPyrénées.

- Luchaire, 1881. "Sur les noms propres basque contenus dans quelques documents pyrénéens des $\mathrm{XI}^{\mathrm{e}}$, $\mathrm{XII}^{\mathrm{e}}$ et $\mathrm{XIII}^{\mathrm{e}}$ siècles". Revue de Linguistique et de Philologie comparée 14, 150-171.

- Malécot, G. (1966). Probabilités et hérédité. Travaux et Documents de l'INED : cahier n47, PUF, Paris.

- Manni, F., E. Guerard, et al. (2004). "Geographic patterns of genetic, morphologic, linguistic variation: how barriers can be detected by using Monmonier's algorithm". Human Biology 76(2): 173-90.

— Michelena, L. (1953). Apellidos vascos, Biblioteca Vascongada de los Amigos del País, San-Sebastián. Réédition, 1977, editorial Txertoa, San-Sebastián.

- Monmonier, M. (1973). Maximum-difference barriers : an alternative numerical regionalization method. Geogr. Anal, 3: 245-61

- Nei, M. (1973). "Genetic distance between populations." American Naturalist 106: 283-292.

— Orpustan, J.B. (1991). Toponymie basque. Presses Universitaires de Bordeaux. Une seconde édition revue et augmentée (2004) est disponible sur l'archive électronique ARTXIKER sous le titre Nouvelle toponymie basque. http://artxiker.ccsd.cnrs.fr.

- Orpustan, J-B. 2000a. Les noms des maisons médiévales en Labourd, BasseNavarre et Soule. Edition Izpegi, Saint-Étienne-de-Baigorry.

- Orpustan, J.B. (2000b). Anthroponomastique médiévale en Pays basque: Prénoms et surnoms en Basse-Navarre et Soule au début du XIV siècle (1305-1350). Lapurdum V, 183-221.

— Oyamburu P. (1991). Euskal Deituren Hiztegia. Édition Hitzak, Ossas-Suhare.

— Pées, A. (2003). Bayonne. Noms de famille \& familles de noms. Atlantica, Bayonne.

— Saitou, N., Nei M. (1987). The neighbor-joining methods : a new method for reconstructing trees. Molecular Biology and Evolution 4(4):406-425.

\section{Remerciements}

Ce travail a été entrepris dans le cadre de l'action CNRS-OHLL (Origine de l'Homme, du Langage et des Langues), programme HIPVAL (History of populations and linguistic variation in Western Pyrenees : Heterogeneity in Basque Populations). Nous tenons également à remercier Franz Manni pour nous avoir initié à son programme BARRIER et pour les nombreux échanges que nous avons eu sur les recherches patronymiques. 


\section{Bigorre}

23 Argelès-Gazost

25 Aucun

26 Bagnères-de-Bigorre

27 La-Barthe-de-Neste

28 Bordères-sur-l'Echez

29 Bordères-Louron

30 Campan

31 Castelnau-Magnoac

32 Castelnau-Rivière-Bas se

33 Galan

34 Lannemezan

35 Luz-Saint-S auveur

36 Maubourguet

37 Mauléon-Barousse

38 Ossun

39 Pouyastruc

40 Rabastens-de-Bigorre

41 Saint-Laurent-de-Neste

42 Saint-Pé-de-Bigorre

43 Tournay

44 Trie-sur-Baïs e

45 Vic-en-Bigorre

46 Vielle-Aure

\section{Pays Basque}

47 La-Bas tide-Cla irence

48 Bidache

49 Espelette

50 Hasparren

51 Hendaye

52 Iholdy

53 Maulé on-Licharre

54 Saint-Etienne-de-Baïgorry

55 Saint-Jean-de-Luz

56 Saint-Je an-Pie d-de-Port

\section{$57 \mathrm{~S}$ aint-Palais}

58 Saint-Pierre-d'Irube

59 Tarde ts

60 Us taritz

Tableau 1 Liste des cantons. Les numéros renvoient à la carte.

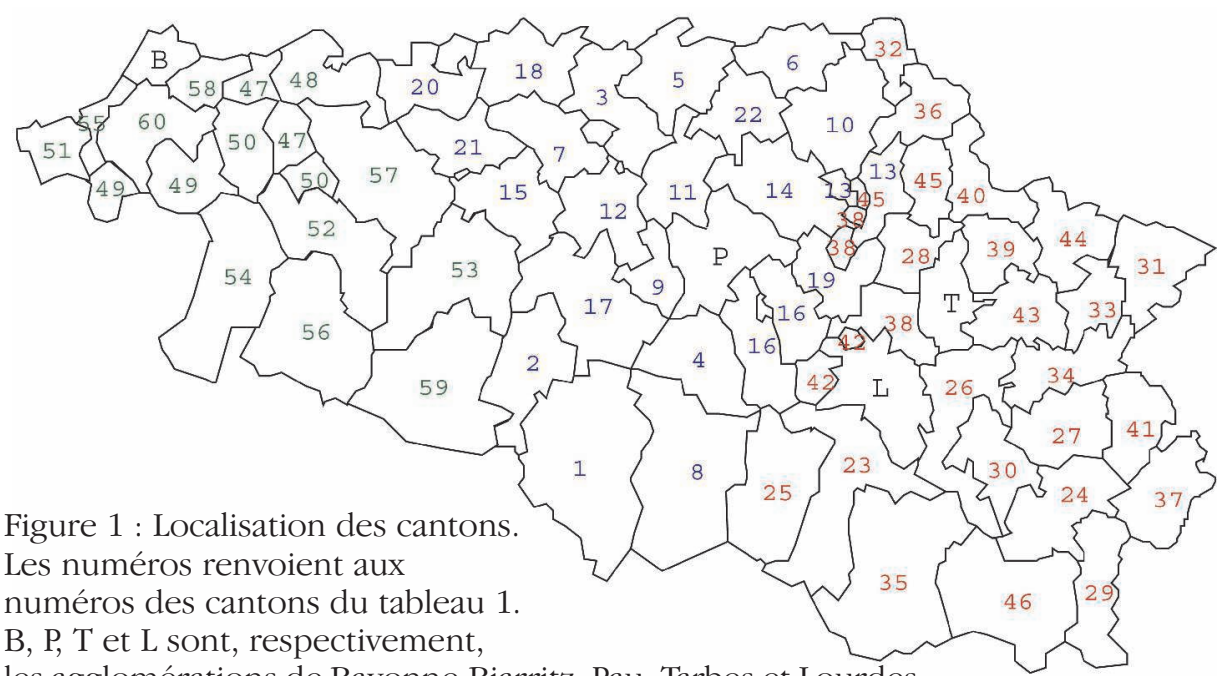
les agglomérations de Bayonne-Biarritz, Pau, Tarbes et Lourdes. 


$\begin{array}{lrccc} & n & S & P & 1000^{*} I \\ \text { Pays Basque } & 14 & 1360(598) & 19.7(4.9) & 0.353(0.127) \\ \text { Béarn } & 22 & 1108(555) & 21.6(3.1) & 0.304(0.085) \\ \text { Bigorre } & 24 & 693(332) & 23.6(6.9) & 0.650(0.324)\end{array}$

Tableau 2 : Moyennes (écarts-type) estimées sur les $n$ cantons de chaque région, du nombre de patronymes, $S$, du potentiel patronymique, $P$ (nombre de patronymes différents pour 100 naissances), et de l'indice d'isonymie $I$ (probabilité de tirer au hasard deux patronymes identiques) (voir texte).

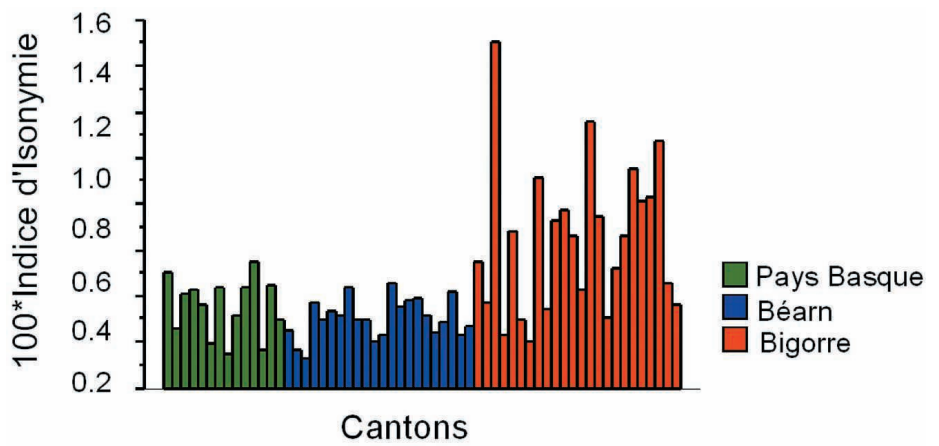

Figure 2a : Distribution des indices d'isonymie par cantons (rangés par ordre alphabétique, voir tableau 1 p. 146) selon leur région d'appartenance.

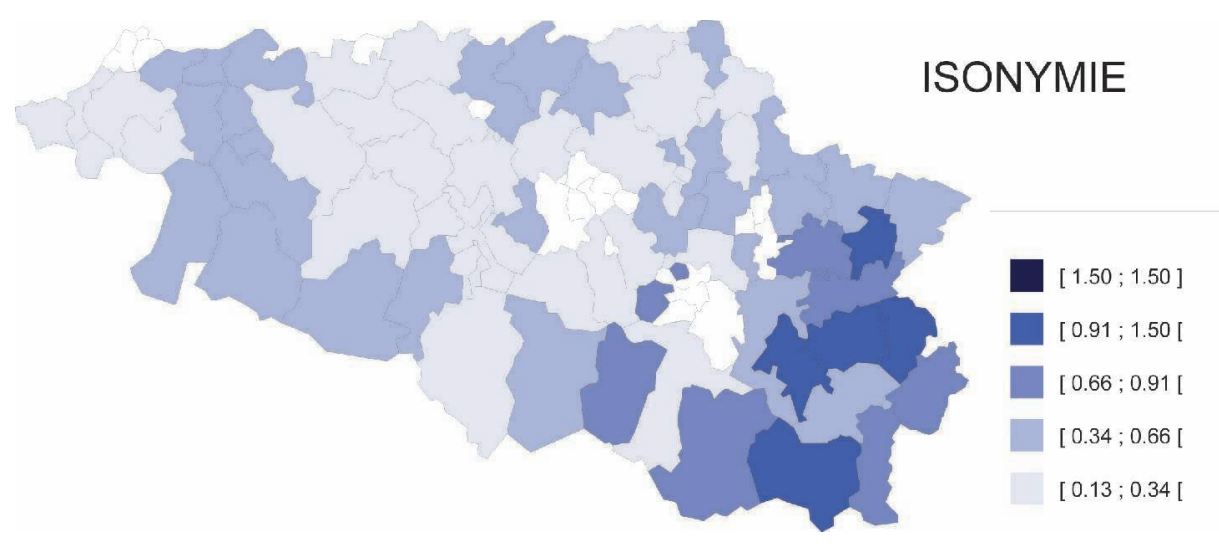

Figure $2 \mathrm{~b}$ : Distribution cantonale du taux d'isonymie (x 1000 ). 


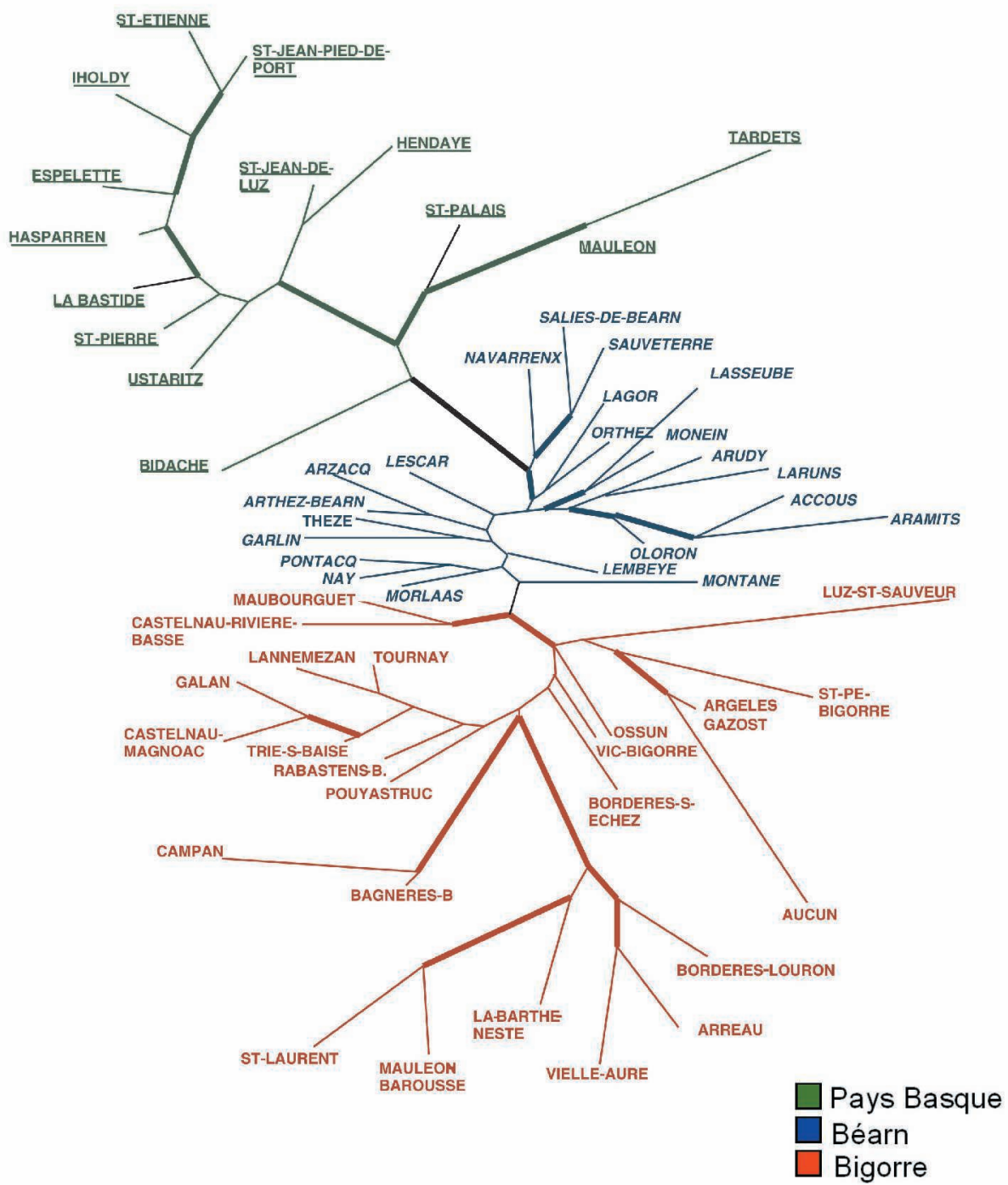

Figure 3. Représentation des distances patronymiques entre cantons par la méthode du Neighbor-joining. Les traits épais délimitent les groupes définis par une valeur de bootstrap supérieure à 90\% (c'est-à-dire les regroupements de cantons les plus robustes). Les cantons basques sont écrits en capitale soulignée, les cantons béarnais en italique et les cantons bigourdans en capitale standard. La longueur des branches indique le degré de différentiation mais leur inclinaison n'a pas de signification particulière. 


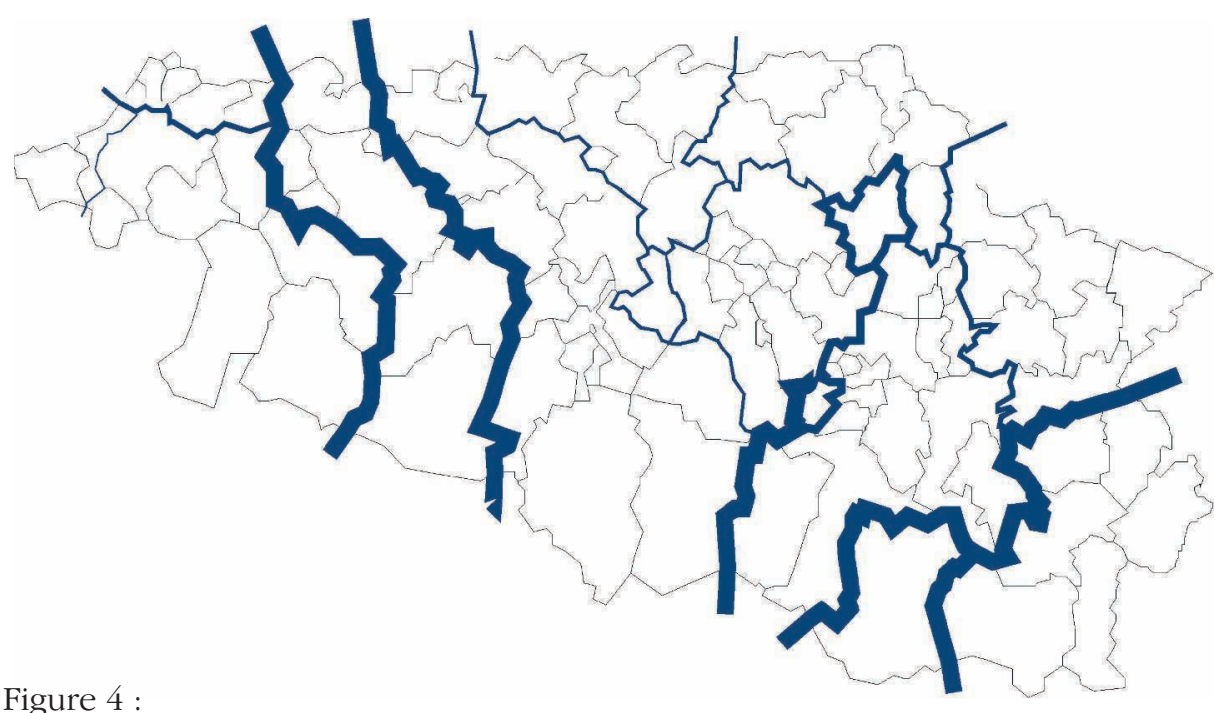

Figure 4:

Localisation des barrières entre cantons

d'après la méthode de Monmonier (Manni et al., 2004).

L'épaisseur des traits est proportionnelle aux valeurs de bootstrap (entre 78\% et $100 \%$ pour le plus épais). Ces barrières délimitent les cantons adjacents dont les distances patronymiques sont les plus fortes (voir texte).

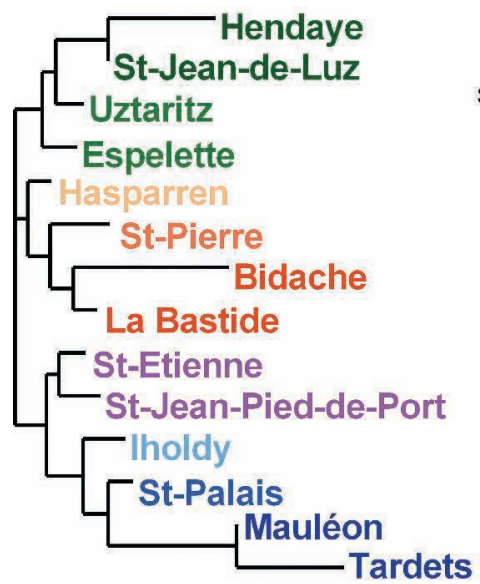

Figure 5a

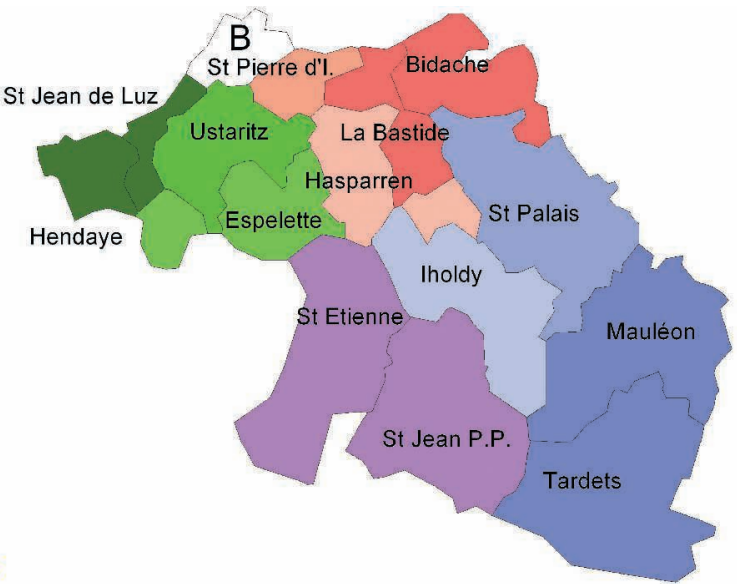

Figure $5 b$

Figure 5: Représentation des distances patronymiques entre cantons du Pays Basque par la méthode du Neighbor-joining (figure 5a). Les groupes sont également projetés dans l'espace géographique (figure 5b) en jouant avec les couleurs et leurs intensités, les cantons de même couleur étant regroupées ensemble. 
Annexe1 : . Pour chaque patronyme et pour chaque région (Pays basque, Béarn et Bigorre) sont indiqués : i) s'il figure dans les 100 (1) ou dans les 200 plus fréquents (2) ; ii) le nombre k de cantons dans lequel au moins une naissance portant ce patronyme a été enregistré, et iii) la fréquence f (multipliée par $10^{3}$ ) du patronyme.

\begin{tabular}{|c|c|c|c|c|c|c|c|}
\hline \multirow[t]{2}{*}{ Patronyme } & \multirow[b]{2}{*}{$\mathbf{i}$} & \multicolumn{2}{|c|}{ Pays Basque } & \multicolumn{2}{|c|}{ Béarn } & \multicolumn{2}{|c|}{ Bigorre } \\
\hline & & $\mathbf{k}$ & $f$ & $\mathbf{k}$ & $\mathbf{f}$ & $\mathbf{k}$ & $f$ \\
\hline$A B A D I E$ & 1 & 12 & 0,879 & 19 & 1,349 & 24 & 16,430 \\
\hline$A B B A D I E$ & 2 & 7 & 0,481 & 7 & 0,316 & 14 & 1,377 \\
\hline$A C C O C E$ & 2 & 3 & 0,722 & 1 & 0,017 & 0 & 0,000 \\
\hline$A G U E R$ & 1 & 5 & 2,678 & 5 & 0,230 & 2 & 0,027 \\
\hline AGUERRE & 1 & 13 & 3,483 & 5 & 0,307 & 1 & 0,014 \\
\hline AGUIRRE & 1 & 9 & 1,674 & 2 & 0,026 & 0 & 0,000 \\
\hline$A M A R E$ & 1 & 0 & 0,000 & 3 & 0,060 & 6 & 2,106 \\
\hline AMESTOY & 1 & 14 & 5,031 & 3 & 0,034 & 0 & 0,000 \\
\hline$A N G L A D E$ & 2 & 2 & 0,126 & 10 & 0,470 & 6 & 1,013 \\
\hline$A R A M B U R U$ & 1 & 6 & 1,297 & 1 & 0,009 & 0 & 0,000 \\
\hline$A R B E R E T$ & 2 & 0 & 0,000 & 0 & 0,000 & 5 & 0,999 \\
\hline$A R L A$ & 2 & 6 & 0,889 & 1 & 0,017 & 0 & 0,000 \\
\hline$A R N E$ & 2 & 0 & 0,000 & 1 & 0,034 & 9 & 1,121 \\
\hline AROSTEGUY & 2 & 6 & 1,119 & 0 & 0,000 & 0 & 0,000 \\
\hline AROTCARENA & 2 & 7 & 0,920 & 0 & 0,000 & 0 & 0,000 \\
\hline ARRETCHE & 2 & 6 & 1,015 & 0 & 0,000 & 0 & 0,000 \\
\hline$A R T O L A$ & 2 & 6 & 0,764 & 1 & 0,094 & 0 & 0,000 \\
\hline$A Y C A G U E R$ & 1 & 11 & 1,349 & 3 & 0,034 & 1 & 0,027 \\
\hline$B A C Q U E$ & 1 & 3 & 0,105 & 16 & 1,152 & 12 & 0,905 \\
\hline$B A D I E$ & 2 & 1 & 0,010 & 8 & 0,743 & 5 & 0,203 \\
\hline$B A R A D A T$ & 1 & 3 & 0,063 & 16 & 2,314 & 2 & 0,041 \\
\hline BARATS & 2 & 0 & 0,000 & 7 & 0,777 & 3 & 0,095 \\
\hline$B A R B A Z A N$ & 2 & 2 & 0,031 & 0 & 0,000 & 9 & 0,891 \\
\hline$B A R B E$ & 1 & 6 & 0,199 & 17 & 1,596 & 17 & 1,296 \\
\hline BAREILLE & 1 & 3 & 0,105 & 9 & 1,144 & 1 & 0,014 \\
\hline BARNECHE & 2 & 8 & 0,805 & 1 & 0,009 & 1 & 0,014 \\
\hline BARNETCHE & 1 & 10 & 1,475 & 0 & 0,000 & 0 & 0,000 \\
\hline$B A R R A Q U E$ & 1 & 3 & 0,042 & 13 & 1,195 & 3 & 0,068 \\
\hline$B A R R E R E$ & 1 & 7 & 0,345 & 15 & 1,315 & 21 & 4,712 \\
\hline BARTHE & 1 & 9 & 0,711 & 14 & 0,768 & 17 & 1,958 \\
\hline$B A Y L A C$ & 1 & 0 & 0,000 & 0 & 0,000 & 8 & 1,485 \\
\hline$B A Y L E$ & 2 & 3 & 0,126 & 14 & 0,785 & 9 & 0,932 \\
\hline$B A Z E R Q U E$ & 1 & 2 & 0,021 & 0 & 0,000 & 12 & 2,484 \\
\hline BEGARIE & 2 & 0 & 0,000 & 3 & 0,128 & 7 & 1,188 \\
\hline$B E G U E$ & 1 & 4 & 0,408 & 14 & 0,657 & 18 & 4,266 \\
\hline BEIGBEDER & 2 & 3 & 0,105 & 13 & 1,033 & 0 & 0,000 \\
\hline$B E L L O C Q$ & 1 & 7 & 0,826 & 20 & 2,220 & 7 & 0,230 \\
\hline$B E R G E$ & 2 & 2 & 0,021 & 13 & 1,042 & 5 & 0,243 \\
\hline BERGERET & 2 & 4 & 0,094 & 11 & 0,896 & 2 & 0,095 \\
\hline BERGES & 1 & 5 & 0,199 & 17 & 1,127 & 6 & 0,216 \\
\hline BERGEZ & 1 & 5 & 0,167 & 16 & 1,938 & 1 & 0,027 \\
\hline BERHO & 1 & 10 & 1,443 & 2 & 0,120 & 0 & 0,000 \\
\hline BERHOUET & 2 & 11 & 1,067 & 0 & 0,000 & 0 & 0,000 \\
\hline BERNET & 2 & 4 & 0,146 & 12 & 0,743 & 6 & 0,338 \\
\hline BERNIGOLE & 2 & 0 & 0,000 & 0 & 0,000 & 2 & 0,986 \\
\hline BEROT & 1 & 1 & 0,042 & 8 & 0,239 & 8 & 1,634 \\
\hline BERROUET & 1 & 7 & 1,151 & 0 & 0,000 & 0 & 0,000 \\
\hline BETBEDER & 2 & 8 & 0,502 & 16 & 0,888 & 1 & 0,014 \\
\hline$B I D A R T$ & 1 & 14 & 5,470 & 7 & 0,179 & 1 & 0,014 \\
\hline BIDEGAIN & 1 & 12 & 2,563 & 1 & 0,009 & 0 & 0,000 \\
\hline BIDEGARAY & 1 & 12 & 1,579 & 2 & 0,017 & 0 & 0,000 \\
\hline BIDONDO & 1 & 6 & 1,433 & 0 & 0,000 & 0 & 0,000 \\
\hline BIS CAY & 1 & 13 & 2,081 & 6 & 0,452 & 1 & 0,014 \\
\hline
\end{tabular}


lapurdum XI 10/12/2007 15:57 Page 153

Distribution des patronymes et structures des populations dans les Pyrénées occidentales

\begin{tabular}{|c|c|c|c|c|c|c|c|}
\hline BONNECAZE & 2 & 1 & 0,021 & 7 & 1,033 & 0 & 0,000 \\
\hline BONNET & 2 & 8 & 0,994 & 10 & 0,316 & 13 & 0,756 \\
\hline BORDA & 1 & 9 & 1,245 & 0 & 0,000 & 0 & 0,000 \\
\hline BORDACHAR & 2 & 3 & 0,732 & 0 & 0,000 & 0 & 0,000 \\
\hline BORDAGARAY & 2 & 5 & 0,952 & 3 & 0,068 & 0 & 0,000 \\
\hline$B O R D E$ & 2 & 1 & 0,010 & 5 & 0,137 & 10 & 1,080 \\
\hline BORDENAVE & 1 & 11 & 0,607 & 22 & 5,472 & 15 & 1,215 \\
\hline BORDES & 1 & 9 & 0,513 & 14 & 1,084 & 15 & 1,526 \\
\hline BOUE & 2 & 3 & 0,073 & 9 & 0,700 & 6 & 0,284 \\
\hline BOURDA & 1 & 4 & 0,052 & 15 & 1,161 & 3 & 0,338 \\
\hline BOURDET & 2 & 5 & 0,094 & 11 & 0,913 & 4 & 0,864 \\
\hline BOURDIEU & 2 & 4 & 0,105 & 15 & 0,931 & 0 & 0,000 \\
\hline BOUS QUET & 1 & 10 & 0,241 & 17 & 1,212 & 11 & 0,837 \\
\hline$B O Y$ & 2 & 2 & 0,042 & 14 & 0,734 & 3 & 0,189 \\
\hline BOYRIE & 2 & 0 & 0,000 & 5 & 0,145 & 6 & 1,188 \\
\hline$B R A N A$ & 2 & 9 & 0,272 & 11 & 0,785 & 0 & 0,000 \\
\hline$B R A U$ & 1 & 1 & 0,021 & 2 & 0,051 & 11 & 2,052 \\
\hline$B R U N E$ & 2 & 2 & 0,021 & 3 & 0,230 & 5 & 0,932 \\
\hline BRUNET & 2 & 0 & 0,000 & 6 & 0,239 & 13 & 1,175 \\
\hline$B R U Z A U D$ & 2 & 0 & 0,000 & 0 & 0,000 & 6 & 1,215 \\
\hline$B U Z Y$ & 2 & 0 & 0,000 & 9 & 0,760 & 2 & 0,122 \\
\hline$C A B A N N E$ & 1 & 5 & 0,439 & 15 & 2,279 & 5 & 0,243 \\
\hline CABARROU & 1 & 0 & 0,000 & 1 & 0,017 & 9 & 2,241 \\
\hline CABOS & 2 & 1 & 0,010 & 1 & 0,009 & 9 & 1,121 \\
\hline$C A M I$ & 2 & 6 & 0,146 & 12 & 0,734 & 3 & 0,081 \\
\hline CAMINO & 2 & 7 & 0,858 & 1 & 0,026 & 0 & 0,000 \\
\hline CAMPAGNE & 1 & 2 & 0,063 & 22 & 2,006 & 2 & 0,122 \\
\hline CAMPAN & 2 & 0 & 0,000 & 1 & 0,017 & 7 & 1,094 \\
\hline$C A M Y$ & 1 & 6 & 0,408 & 17 & 1,810 & 6 & 0,176 \\
\hline$C A N D A U$ & 1 & 5 & 0,544 & 15 & 1,426 & 3 & 0,054 \\
\hline CANTON & 1 & 4 & 0,126 & 16 & 1,289 & 5 & 0,419 \\
\hline CAPDEPON & 2 & 1 & 0,052 & 9 & 0,905 & 0 & 0,000 \\
\hline CAPDEVIELLE & 1 & 10 & 0,293 & 21 & 3,432 & 11 & 1,620 \\
\hline CAPDEVILLE & 2 & 10 & 0,805 & 14 & 0,632 & 11 & 0,662 \\
\hline CARASSUS & 2 & 0 & 0,000 & 4 & 0,120 & 9 & 1,013 \\
\hline$C A R R E R E$ & 1 & 11 & 0,837 & 18 & 3,918 & 24 & 8,505 \\
\hline$C A R R E Y$ & 2 & 1 & 0,021 & 9 & 0,837 & 0 & 0,000 \\
\hline CARRICABURU & 1 & 9 & 1,422 & 2 & 0,026 & 0 & 0,000 \\
\hline CASABONNE & 2 & 4 & 0,157 & 6 & 0,785 & 1 & 0,014 \\
\hline CASAMAYOU & 2 & 3 & 0,073 & 10 & 0,802 & 1 & 0,014 \\
\hline CASANAVE & 2 & 2 & 0,021 & 13 & 0,956 & 0 & 0,000 \\
\hline CASASSUS & 2 & 2 & 0,021 & 5 & 0,871 & 1 & 0,014 \\
\hline$C A S A U B O N$ & 2 & 8 & 0,251 & 11 & 1,033 & 0 & 0,000 \\
\hline CASAUX & 1 & 4 & 0,282 & 9 & 1,161 & 1 & 0,014 \\
\hline CASENAVE & 1 & 12 & 0,879 & 17 & 2,015 & 0 & 0,000 \\
\hline CASSOU & 1 & 6 & 0,251 & 19 & 3,850 & 9 & 0,837 \\
\hline CASTAGNET & 2 & 7 & 0,272 & 14 & 1,084 & 4 & 0,162 \\
\hline CAS TAING & 1 & 5 & 0,084 & 14 & 1,323 & 16 & 1,013 \\
\hline CASTERA & 1 & 4 & 0,282 & 14 & 1,537 & 5 & 0,162 \\
\hline CASTERAN & 1 & 0 & 0,000 & 3 & 0,034 & 13 & 5,171 \\
\hline CASTET & 1 & 5 & 0,345 & 7 & 0,470 & 14 & 1,566 \\
\hline CASTETS & 1 & 3 & 0,115 & 6 & 0,196 & 15 & 1,512 \\
\hline CASTEX & 2 & 1 & 0,021 & 3 & 0,043 & 8 & 0,959 \\
\hline САUHAPE & 1 & 0 & 0,000 & 13 & 1,392 & 3 & 0,108 \\
\hline CAUS SADE & 2 & 3 & 0,094 & 3 & 0,060 & 10 & 1,053 \\
\hline$C A Z A B A N$ & 1 & 1 & 0,010 & 12 & 1,596 & 8 & 0,216 \\
\hline
\end{tabular}




\begin{tabular}{|c|c|c|c|c|c|c|c|}
\hline$C A Z A B A T$ & 2 & 0 & 0,000 & 4 & 0,137 & 11 & 1,121 \\
\hline CAZAJOUS & 1 & 2 & 0,021 & 4 & 0,282 & 5 & 1,877 \\
\hline$C A Z A L A$ & 2 & 2 & 0,031 & 10 & 0,700 & 4 & 0,405 \\
\hline CAZALAS & 1 & 0 & 0,000 & 0 & 0,000 & 11 & 1,620 \\
\hline CAZALIS & 2 & 4 & 0,136 & 11 & 0,811 & 2 & 0,068 \\
\hline$C A Z A N A V E$ & 2 & 2 & 0,042 & 12 & 0,666 & 8 & 0,540 \\
\hline CAZAUBON & 2 & 4 & 0,136 & 11 & 0,768 & 14 & 1,215 \\
\hline CAZAUX & 1 & 11 & 0,795 & 19 & 1,306 & 21 & 4,482 \\
\hline CAZENAVE & 1 & 14 & 0,910 & 21 & 7,034 & 19 & 4,239 \\
\hline CAZES & 2 & 1 & 0,010 & 2 & 0,017 & 12 & 1,202 \\
\hline CHAGUE & 1 & 3 & 0,073 & 8 & 1,554 & 2 & 0,068 \\
\hline $\mathrm{CHOY}$ & 2 & 2 & 0,063 & 8 & 0,802 & 0 & 0,000 \\
\hline CIEUTAT & 1 & 0 & 0,000 & 0 & 0,000 & 9 & 2,241 \\
\hline CLARENS & 2 & 1 & 0,010 & 1 & 0,009 & 9 & 1,242 \\
\hline CLAVERIE & 1 & 13 & 1,475 & 22 & 3,936 & 22 & 4,145 \\
\hline$C L O S$ & 2 & 1 & 0,010 & 8 & 0,879 & 9 & 0,621 \\
\hline COLOMES & 2 & 0 & 0,000 & 0 & 0,000 & 9 & 1,269 \\
\hline COMMERES & 2 & 0 & 0,000 & 0 & 0,000 & 10 & 0,986 \\
\hline COMPAGNET & 2 & 0 & 0,000 & 0 & 0,000 & 7 & 1,080 \\
\hline CORREGE & 1 & 0 & 0,000 & 1 & 0,009 & 10 & 2,079 \\
\hline COS TEDOAT & 2 & 1 & 0,010 & 7 & 0,956 & 0 & 0,000 \\
\hline COUGET & 1 & 2 & 0,031 & 3 & 0,043 & 15 & 1,688 \\
\hline COURREGES & 1 & 1 & 0,010 & 16 & 1,818 & 9 & 0,878 \\
\hline COURTADE & 1 & 3 & 0,084 & 10 & 0,410 & 14 & 1,647 \\
\hline COUS SIRAT & 2 & 1 & 0,021 & 7 & 0,640 & 0 & 0,000 \\
\hline COUSTET & 2 & 0 & 0,000 & 11 & 0,649 & 1 & 0,041 \\
\hline$C R A M P E$ & 1 & 1 & 0,010 & 2 & 0,094 & 8 & 1,607 \\
\hline CUILHE & 2 & 0 & 0,000 & 2 & 0,034 & 8 & 0,918 \\
\hline CURUTCHET & 1 & 14 & 4,152 & 0 & 0,000 & 0 & 0,000 \\
\hline$D A B A T$ & 1 & 0 & 0,000 & 3 & 0,085 & 8 & 1,877 \\
\hline$D A B B A D I E$ & 2 & 8 & 0,795 & 4 & 0,171 & 0 & 0,000 \\
\hline$D A C H A R Y$ & 1 & 10 & 1,862 & 5 & 0,239 & 0 & 0,000 \\
\hline$D A G U E R R E$ & 1 & 14 & 3,002 & 6 & 0,410 & 1 & 0,014 \\
\hline DAMESTOY & 1 & 11 & 1,297 & 1 & 0,009 & 0 & 0,000 \\
\hline$D A R R A C Q$ & 1 & 3 & 0,042 & 12 & 1,340 & 0 & 0,000 \\
\hline$D A R R E$ & 1 & 0 & 0,000 & 4 & 0,171 & 14 & 2,106 \\
\hline DARRIEUMERLOU & 1 & 9 & 1,569 & 2 & 0,077 & 1 & 0,014 \\
\hline$D A R R I G R A N D$ & 2 & 2 & 0,126 & 8 & 0,965 & 1 & 0,027 \\
\hline$D A S Q U E$ & 2 & 0 & 0,000 & 1 & 0,060 & 11 & 1,323 \\
\hline DASTUGUE & 1 & 0 & 0,000 & 1 & 0,009 & 9 & 1,958 \\
\hline$D A Z E T$ & 2 & 0 & 0,000 & 2 & 0,017 & 9 & 0,986 \\
\hline$D E L A S$ & 2 & 3 & 0,115 & 1 & 0,034 & 9 & 1,364 \\
\hline DES PIAU & 2 & 0 & 0,000 & 0 & 0,000 & 5 & 1,350 \\
\hline DOMEC & 1 & 3 & 0,439 & 4 & 0,043 & 15 & 1,958 \\
\hline$D O M E C Q$ & 1 & 5 & 0,282 & 15 & 1,383 & 3 & 0,068 \\
\hline$D O M E R C Q$ & 2 & 1 & 0,010 & 5 & 0,649 & 0 & 0,000 \\
\hline DOS PITAL & 2 & 9 & 0,753 & 0 & 0,000 & 0 & 0,000 \\
\hline DOUX & 2 & 1 & 0,010 & 3 & 0,120 & 10 & 1,215 \\
\hline DOYHENARD & 2 & 7 & 0,784 & 0 & 0,000 & 0 & 0,000 \\
\hline DUBARRY & 1 & 1 & 0,010 & 2 & 0,034 & 17 & 5,144 \\
\hline$D U B A U$ & 1 & 0 & 0,000 & 1 & 0,009 & 6 & 1,472 \\
\hline$D U B O E$ & 1 & 0 & 0,000 & 2 & 0,128 & 7 & 1,566 \\
\hline DUBOSC & 1 & 0 & 0,000 & 4 & 0,137 & 8 & 1,472 \\
\hline DUCASSE & 1 & 7 & 0,377 & 9 & 0,717 & 16 & 2,295 \\
\hline DUCLOS & 1 & 5 & 0,282 & 10 & 0,478 & 16 & 2,849 \\
\hline DUCOS & 2 & 3 & 0,084 & 14 & 0,956 & 12 & 1,215 \\
\hline
\end{tabular}


lapurdum XI 10/12/2007 15:57 Page 155

\begin{tabular}{|c|c|c|c|c|c|c|c|}
\hline DUCUING & 2 & 0 & 0,000 & 1 & 0,009 & 8 & 1,269 \\
\hline$D U F A U$ & 1 & 13 & 1,904 & 18 & 2,442 & 4 & 0,068 \\
\hline$D U F F A U$ & 1 & 1 & 0,031 & 6 & 0,137 & 18 & 2,714 \\
\hline$D U F F O$ & 2 & 0 & 0,000 & 0 & 0,000 & 9 & 1,323 \\
\hline DUFOURCQ & 1 & 8 & 0,136 & 11 & 1,229 & 1 & 0,014 \\
\hline DUHALDE & 1 & 12 & 3,033 & 2 & 0,026 & 0 & 0,000 \\
\hline DUHART & 1 & 9 & 2,040 & 0 & 0,000 & 0 & 0,000 \\
\hline$D U H A U$ & 1 & 12 & 1,443 & 6 & 0,188 & 1 & 0,014 \\
\hline DULOUT & 1 & 1 & 0,021 & 2 & 0,017 & 10 & 1,634 \\
\hline DUMESTRE & 2 & 0 & 0,000 & 0 & 0,000 & 10 & 1,040 \\
\hline$D U P L A A$ & 2 & 6 & 0,199 & 10 & 0,990 & 1 & 0,027 \\
\hline$D U P L A N$ & 2 & 0 & 0,000 & 1 & 0,017 & 10 & 1,391 \\
\hline DUPONT & 1 & 5 & 0,052 & 11 & 0,427 & 19 & 3,335 \\
\hline DUPOUY & 1 & 7 & 0,429 & 10 & 1,238 & 14 & 0,662 \\
\hline DUPRAT & 1 & 5 & 0,094 & 5 & 0,213 & 15 & 2,174 \\
\hline$D U P U Y$ & 1 & 7 & 0,272 & 17 & 0,427 & 18 & 3,578 \\
\hline DURRUTY & 1 & 9 & 1,789 & 1 & 0,009 & 0 & 0,000 \\
\hline DUSSERT & 2 & 0 & 0,000 & 0 & 0,000 & 8 & 1,391 \\
\hline DUTHU & 1 & 1 & 0,031 & 0 & 0,000 & 15 & 3,443 \\
\hline DUTREY & 1 & 2 & 0,105 & 1 & 0,009 & 12 & 1,472 \\
\hline ECHEVERRIA & 1 & 7 & 1,778 & 0 & 0,000 & 1 & 0,027 \\
\hline ECHEVESTE & 2 & 4 & 0,795 & 0 & 0,000 & 0 & 0,000 \\
\hline ELGART & 1 & 9 & 1,140 & 0 & 0,000 & 0 & 0,000 \\
\hline ELIS SALDE & 1 & 11 & 4,058 & 0 & 0,000 & 2 & 0,068 \\
\hline ELIZALDE & 2 & 7 & 0,743 & 1 & 0,009 & 0 & 0,000 \\
\hline ERRAMOUSPE & 2 & 6 & 1,015 & 1 & 0,009 & 0 & 0,000 \\
\hline ERRANDONEA & 2 & 4 & 1,109 & 0 & 0,000 & 0 & 0,000 \\
\hline ERRECART & 1 & 12 & 2,155 & 3 & 0,051 & 0 & 0,000 \\
\hline ESPIL & 2 & 7 & 0,889 & 1 & 0,009 & 1 & 0,014 \\
\hline ESQUERRE & 2 & 0 & 0,000 & 7 & 0,598 & 11 & 0,891 \\
\hline ESTRADE & 1 & 1 & 0,010 & 6 & 0,239 & 11 & 1,404 \\
\hline ETCHART & 1 & 13 & 4,770 & 2 & 0,017 & 0 & 0,000 \\
\hline ETCHEBARNE & 1 & 13 & 3,598 & 4 & 0,188 & 0 & 0,000 \\
\hline ETCHEBER & 2 & 3 & 0,711 & 2 & 0,051 & 0 & 0,000 \\
\hline ETCHEBERRY & 1 & 14 & 4,372 & 4 & 0,154 & 0 & 0,000 \\
\hline ETCHEBEST & 2 & 7 & 0,753 & 1 & 0,026 & 0 & 0,000 \\
\hline ETCHECHOURY & 2 & 11 & 0,711 & 0 & 0,000 & 0 & 0,000 \\
\hline ETCHECOPAR & 1 & 7 & 2,113 & 1 & 0,009 & 0 & 0,000 \\
\hline ETCHEGARAY & 1 & 14 & 4,811 & 0 & 0,000 & 0 & 0,000 \\
\hline ETCHEGOIN & 1 & 7 & 1,318 & 1 & 0,017 & 0 & 0,000 \\
\hline ETCHEGOYEN & 1 & 13 & 1,757 & 2 & 0,026 & 0 & 0,000 \\
\hline ETCHEGOYHEN & 2 & 3 & 1,025 & 3 & 0,077 & 0 & 0,000 \\
\hline ETCHEMENDY & 1 & 9 & 1,778 & 0 & 0,000 & 0 & 0,000 \\
\hline ETCHENIQUE & 2 & 6 & 0,816 & 2 & 0,017 & 1 & 0,014 \\
\hline ETCHEPARE & 1 & 13 & 2,636 & 3 & 0,068 & 0 & 0,000 \\
\hline ETCHETO & 1 & 13 & 1,412 & 0 & 0,000 & 1 & 0,014 \\
\hline ETCHEVERRIA & 1 & 12 & 2,196 & 5 & 0,043 & 0 & 0,000 \\
\hline ETCHEVERRY & 1 & 14 & 16,798 & 9 & 0,521 & 1 & 0,014 \\
\hline ETCHEVERS & 1 & 12 & 1,255 & 2 & 0,017 & 0 & 0,000 \\
\hline EYHERABIDE & 1 & 9 & 1,255 & 0 & 0,000 & 1 & 0,027 \\
\hline EYHERAMENDY & 2 & 6 & 0,764 & 1 & 0,043 & 0 & 0,000 \\
\hline FAGOAGA & 2 & 4 & 0,784 & 0 & 0,000 & 0 & 0,000 \\
\hline FERNANDEZ & 2 & 10 & 0,784 & 10 & 0,589 & 9 & 0,297 \\
\hline FERRAN & 2 & 0 & 0,000 & 6 & 0,350 & 12 & 0,905 \\
\hline FEUGAS & 2 & 0 & 0,000 & 12 & 1,033 & 0 & 0,000 \\
\hline FONTAN & 1 & 3 & 0,209 & 8 & 0,290 & 22 & 4,361 \\
\hline
\end{tabular}




\begin{tabular}{|c|c|c|c|c|c|c|c|}
\hline FORGUE & 2 & 0 & 0,000 & 1 & 0,017 & 10 & 1,377 \\
\hline FORGUES & 2 & 2 & 0,021 & 6 & 0,171 & 14 & 1,310 \\
\hline FOURCADE & 1 & 11 & 0,868 & 18 & 2,006 & 23 & 9,869 \\
\hline FOURQUET & 1 & 1 & 0,010 & 3 & 0,171 & 8 & 1,620 \\
\hline FOURTINE & 2 & 0 & 0,000 & 0 & 0,000 & 4 & 1,067 \\
\hline FRANCHIS TEGUY & 2 & 2 & 0,722 & 0 & 0,000 & 0 & 0,000 \\
\hline FRECHOU & 2 & 4 & 0,105 & 11 & 0,427 & 13 & 1,080 \\
\hline GACHAS SIN & 2 & 0 & 0,000 & 0 & 0,000 & 9 & 0,918 \\
\hline GACHEN & 2 & 8 & 0,900 & 2 & 0,060 & 0 & 0,000 \\
\hline$G A L A N$ & 2 & 1 & 0,031 & 7 & 0,546 & 8 & 1,161 \\
\hline GALIAY & 2 & 1 & 0,010 & 1 & 0,009 & 6 & 0,905 \\
\hline$G A R A T$ & 1 & 14 & 3,410 & 8 & 0,470 & 0 & 0,000 \\
\hline GARAY & 2 & 8 & 0,795 & 1 & 0,017 & 1 & 0,041 \\
\hline GARCIA & 1 & 10 & 1,809 & 10 & 1,050 & 11 & 0,837 \\
\hline GARMENDIA & 2 & 6 & 0,858 & 1 & 0,009 & 0 & 0,000 \\
\hline GAUBERT & 2 & 0 & 0,000 & 1 & 0,009 & 5 & 1,013 \\
\hline$G A Y E$ & 1 & 3 & 0,094 & 8 & 0,418 & 14 & 2,349 \\
\hline GONI & 2 & 9 & 0,858 & 1 & 0,017 & 0 & 0,000 \\
\hline GOYA & 2 & 5 & 0,732 & 0 & 0,000 & 0 & 0,000 \\
\hline GOYHENETCHE & 2 & 7 & 0,784 & 0 & 0,000 & 0 & 0,000 \\
\hline GRACIA & 2 & 3 & 0,105 & 8 & 0,837 & 4 & 0,351 \\
\hline GRANGE & 2 & 4 & 0,094 & 7 & 0,922 & 8 & 0,689 \\
\hline GUINLE & 2 & 0 & 0,000 & 0 & 0,000 & 8 & 0,972 \\
\hline$H A G E T$ & 2 & 2 & 0,105 & 12 & 0,820 & 3 & 0,054 \\
\hline HARAMBOURE & 1 & 9 & 1,360 & 0 & 0,000 & 0 & 0,000 \\
\hline$H A R A N$ & 2 & 9 & 1,046 & 0 & 0,000 & 0 & 0,000 \\
\hline HARGUINDEGUY & 1 & 12 & 2,542 & 0 & 0,000 & 0 & 0,000 \\
\hline HAR IS MENDY & 1 & 10 & 1,339 & 2 & 0,051 & 2 & 0,054 \\
\hline HARIS PE & 2 & 11 & 0,764 & 0 & 0,000 & 0 & 0,000 \\
\hline HARISTOY & 1 & 11 & 1,590 & 2 & 0,026 & 0 & 0,000 \\
\hline HARRIET & 1 & 9 & 1,171 & 0 & 0,000 & 0 & 0,000 \\
\hline HASTOY & 2 & 9 & 0,826 & 0 & 0,000 & 0 & 0,000 \\
\hline$H A U$ & 2 & 0 & 0,000 & 11 & 0,649 & 2 & 0,027 \\
\hline HAURE & 2 & 1 & 0,010 & 14 & 0,734 & 6 & 0,203 \\
\hline HAURET & 2 & 4 & 0,073 & 10 & 0,973 & 3 & 0,189 \\
\hline HAURIE & 2 & 3 & 0,335 & 10 & 0,922 & 0 & 0,000 \\
\hline HAURINE & 2 & 1 & 0,010 & 0 & 0,000 & 2 & 0,891 \\
\hline HAYET & 2 & 9 & 0,952 & 5 & 0,154 & 0 & 0,000 \\
\hline HEGOBURU & 1 & 4 & 1,234 & 2 & 0,017 & 0 & 0,000 \\
\hline$H E G U Y$ & 1 & 11 & 1,736 & 0 & 0,000 & 2 & 0,027 \\
\hline HIR IART & 1 & 13 & 3,744 & 2 & 0,017 & 0 & 0,000 \\
\hline HIR IBARREN & 1 & 5 & 1,151 & 0 & 0,000 & 0 & 0,000 \\
\hline HIRIGOYEN & 1 & 12 & 4,006 & 2 & 0,077 & 0 & 0,000 \\
\hline HOURCADE & 1 & 7 & 1,140 & 19 & 2,587 & 5 & 0,365 \\
\hline HOURDEBAIGT & 1 & 2 & 0,031 & 7 & 1,195 & 0 & 0,000 \\
\hline$I B A R R A$ & 2 & 8 & 0,774 & 1 & 0,009 & 0 & 0,000 \\
\hline$I B O S$ & 1 & 1 & 0,010 & 2 & 0,034 & 10 & 2,187 \\
\hline IDIART & 1 & 11 & 3,117 & 2 & 0,034 & 0 & 0,000 \\
\hline INCHAUS PE & 1 & 10 & 1,600 & 1 & 0,009 & 0 & 0,000 \\
\hline$I N D A$ & 2 & 7 & 0,952 & 0 & 0,000 & 0 & 0,000 \\
\hline$I N D A R T$ & 1 & 7 & 1,287 & 0 & 0,000 & 0 & 0,000 \\
\hline IRASTORZA & 2 & 4 & 0,722 & 0 & 0,000 & 0 & 0,000 \\
\hline IRATCABAL & 2 & 6 & 1,046 & 1 & 0,009 & 0 & 0,000 \\
\hline $\operatorname{IRIART}$ & 2 & 9 & 2,814 & 2 & 0,128 & 0 & 0,000 \\
\hline IRIARTE & 2 & 7 & 0,826 & 1 & 0,085 & 0 & 0,000 \\
\hline IRIBARNE & 1 & 11 & 2,385 & 0 & 0,000 & 0 & 0,000 \\
\hline
\end{tabular}




\begin{tabular}{|c|c|c|c|c|c|c|c|}
\hline IRIBARREN & 1 & 11 & 1,569 & 1 & 0,009 & 0 & 0,000 \\
\hline IRIBERRY & 2 & 5 & 0,868 & 1 & 0,017 & 0 & 0,000 \\
\hline IRIGARAY & 1 & 7 & 2,008 & 0 & 0,000 & 0 & 0,000 \\
\hline IRIGOIN & 1 & 8 & 1,506 & 1 & 0,017 & 0 & 0,000 \\
\hline IRIGOYEN & 1 & 10 & 2,406 & 2 & 0,043 & 1 & 0,041 \\
\hline ITHURBIDE & 2 & 11 & 1,088 & 1 & 0,017 & 0 & 0,000 \\
\hline ITHURRALDE & 1 & 7 & 1,297 & 1 & 0,009 & 1 & 0,027 \\
\hline ITURRIA & 2 & 7 & 1,004 & 0 & 0,000 & 0 & 0,000 \\
\hline JAUREGUIBERRY & 1 & 11 & 2,594 & 2 & 0,060 & 0 & 0,000 \\
\hline JAUREGUY & 1 & 12 & 1,496 & 3 & 0,043 & 1 & 0,014 \\
\hline JUNCA & 2 & 5 & 0,105 & 8 & 0,487 & 13 & 0,972 \\
\hline$L A B A N$ & 2 & 1 & 0,010 & 8 & 0,717 & 7 & 0,459 \\
\hline LABARRERE & 1 & 4 & 0,146 & 15 & 1,152 & 7 & 0,581 \\
\hline LABARTHE & 1 & 11 & 0,648 & 19 & 2,092 & 4 & 0,243 \\
\hline$L A B A S T I E$ & 2 & 6 & 0,241 & 9 & 0,837 & 0 & 0,000 \\
\hline$L A B A T$ & 1 & 12 & 2,583 & 20 & 2,740 & 15 & 2,133 \\
\hline$L A B I T$ & 1 & 0 & 0,000 & 1 & 0,043 & 4 & 1,445 \\
\hline$\angle A B O R D E$ & 1 & 14 & 3,525 & 22 & 10,449 & 15 & 2,039 \\
\hline LABOURDETTE & 1 & 5 & 0,157 & 19 & 3,090 & 3 & 0,162 \\
\hline$L A C A B A N N E$ & 2 & 0 & 0,000 & 14 & 0,666 & 3 & 0,095 \\
\hline$L A C A U$ & 2 & 2 & 0,073 & 8 & 0,666 & 3 & 0,311 \\
\hline$L A C A Z E$ & 1 & 8 & 0,282 & 18 & 1,571 & 20 & 3,672 \\
\hline$L A C L A U$ & 1 & 8 & 0,293 & 13 & 1,468 & 0 & 0,000 \\
\hline LACOME & 2 & 1 & 0,010 & 2 & 0,017 & 5 & 0,959 \\
\hline LACOSTE & 1 & 13 & 0,722 & 20 & 3,756 & 20 & 3,227 \\
\hline$L A C R A M P E$ & 1 & 2 & 0,021 & 10 & 1,238 & 15 & 2,066 \\
\hline LACROIX & 1 & 12 & 1,192 & 13 & 0,538 & 10 & 0,405 \\
\hline LACROUTS & 1 & 4 & 0,199 & 17 & 1,417 & 6 & 0,216 \\
\hline$L A F A R G U E$ & 1 & 6 & 0,795 & 18 & 1,520 & 8 & 0,203 \\
\hline LAFFAILLE & 1 & 0 & 0,000 & 2 & 0,102 & 6 & 2,741 \\
\hline LAFFITTE & 1 & 12 & 0,900 & 14 & 1,246 & 11 & 0,567 \\
\hline LAFFONT & 1 & 1 & 0,010 & 4 & 0,102 & 14 & 1,607 \\
\hline$L A F F O R G U E$ & 1 & 0 & 0,000 & 3 & 0,171 & 17 & 2,660 \\
\hline LAFITTE & 1 & 12 & 2,500 & 15 & 1,528 & 5 & 0,162 \\
\hline$L A F O N$ & 2 & 1 & 0,010 & 18 & 1,007 & 3 & 0,216 \\
\hline$L A F O N T$ & 2 & 5 & 0,126 & 12 & 0,760 & 6 & 0,176 \\
\hline LAFOURCADE & 1 & 9 & 0,784 & 15 & 1,204 & 3 & 0,203 \\
\hline$L A G A R D E$ & 1 & 8 & 0,335 & 14 & 0,922 & 10 & 0,662 \\
\hline LAGRAVE & 2 & 0 & 0,000 & 13 & 1,152 & 4 & 0,176 \\
\hline LAHAILLE & 2 & 0 & 0,000 & 6 & 0,196 & 11 & 0,986 \\
\hline LAHITTE & 1 & 4 & 0,157 & 16 & 1,093 & 1 & 0,014 \\
\hline LAHON & 2 & 1 & 0,010 & 9 & 0,751 & 3 & 0,095 \\
\hline LAHORE & 2 & 1 & 0,021 & 11 & 0,999 & 4 & 0,243 \\
\hline LAJUS & 2 & 3 & 0,063 & 9 & 0,640 & 4 & 0,176 \\
\hline$L A L A N N E$ & 1 & 10 & 0,450 & 22 & 5,814 & 13 & 1,917 \\
\hline$L A M A R Q U E$ & 1 & 6 & 0,690 & 19 & 2,365 & 13 & 1,431 \\
\hline$\angle A M A Z O U$ & 2 & 1 & 0,010 & 10 & 0,640 & 0 & 0,000 \\
\hline LAMOTHE & 2 & 9 & 0,858 & 14 & 0,760 & 5 & 0,297 \\
\hline LANNE & 2 & 0 & 0,000 & 12 & 0,896 & 5 & 0,392 \\
\hline LANNES & 2 & 1 & 0,031 & 15 & 0,691 & 6 & 0,581 \\
\hline$\angle A N O T$ & 2 & 1 & 0,010 & 6 & 0,743 & 2 & 0,027 \\
\hline$\angle A N S A L O T$ & 2 & 2 & 0,052 & 8 & 0,837 & 3 & 0,081 \\
\hline LANUS SE & 2 & 4 & 0,220 & 13 & 1,033 & 6 & 0,621 \\
\hline LAPEYRE & 1 & 7 & 0,335 & 8 & 0,905 & 16 & 1,796 \\
\hline LAPLACE & 1 & 9 & 0,554 & 22 & 2,954 & 8 & 0,486 \\
\hline LAPORTE & 1 & 9 & 0,586 & 18 & 1,246 & 17 & 2,930 \\
\hline
\end{tabular}




\begin{tabular}{|c|c|c|c|c|c|c|c|}
\hline$L A P U Y A D E$ & 2 & 1 & 0,105 & 13 & 0,845 & 3 & 0,095 \\
\hline$L A R A N$ & 1 & 0 & 0,000 & 0 & 0,000 & 7 & 1,620 \\
\hline LARQUIER & 2 & 1 & 0,010 & 10 & 0,990 & 3 & 0,054 \\
\hline$L A R R A L D E$ & 1 & 13 & 2,552 & 2 & 0,017 & 1 & 0,014 \\
\hline LARRAMENDY & 1 & 11 & 2,385 & 4 & 0,051 & 0 & 0,000 \\
\hline$L A R R E$ & 1 & 13 & 4,236 & 11 & 0,700 & 13 & 2,147 \\
\hline LARREGAIN & 1 & 12 & 1,213 & 5 & 0,102 & 1 & 0,014 \\
\hline$L A R R I E U$ & 1 & 8 & 0,293 & 22 & 3,620 & 17 & 1,175 \\
\hline$L A R R O N D E$ & 1 & 8 & 1,653 & 0 & 0,000 & 1 & 0,014 \\
\hline LARRONDO & 2 & 9 & 0,983 & 1 & 0,009 & 0 & 0,000 \\
\hline$L A R R O Q U E$ & 1 & 6 & 0,732 & 16 & 1,144 & 11 & 0,513 \\
\hline$L A R R O U D E$ & 2 & 6 & 0,293 & 14 & 0,649 & 6 & 0,297 \\
\hline LARROUY & 1 & 3 & 0,157 & 19 & 1,426 & 11 & 0,864 \\
\hline$L A R T I G A U$ & 2 & 9 & 0,554 & 14 & 0,657 & 0 & 0,000 \\
\hline$L A R T I G U E$ & 1 & 10 & 0,743 & 15 & 1,366 & 10 & 0,932 \\
\hline$L A R Z A B A L$ & 1 & 5 & 1,360 & 0 & 0,000 & 0 & 0,000 \\
\hline LASCARAY & 2 & 3 & 0,711 & 0 & 0,000 & 0 & 0,000 \\
\hline$L A S S A L L E$ & 1 & 12 & 0,784 & 22 & 4,047 & 10 & 0,810 \\
\hline LASS ERRE & 1 & 11 & 0,910 & 22 & 2,433 & 14 & 1,215 \\
\hline$L A S S U S$ & 1 & 6 & 0,241 & 17 & 2,023 & 13 & 1,377 \\
\hline LATAILLADE & 2 & 9 & 0,920 & 6 & 0,282 & 1 & 0,014 \\
\hline LATAPIE & 1 & 3 & 0,126 & 15 & 1,059 & 16 & 3,281 \\
\hline LATOUR & 1 & 4 & 0,199 & 8 & 0,154 & 15 & 1,904 \\
\hline LATRUBESSE & 2 & 1 & 0,031 & 7 & 0,794 & 1 & 0,014 \\
\hline$L A U G A$ & 1 & 3 & 0,094 & 16 & 1,263 & 3 & 0,122 \\
\hline LAULHE & 1 & 4 & 0,094 & 14 & 1,707 & 2 & 0,027 \\
\hline$L A V I E$ & 1 & 10 & 0,607 & 15 & 1,528 & 1 & 0,014 \\
\hline LAVIGNASSE & 2 & 7 & 0,753 & 5 & 0,120 & 0 & 0,000 \\
\hline$L A V I G N E$ & 1 & 9 & 0,732 & 19 & 2,390 & 15 & 1,701 \\
\hline LAVIT & 2 & 0 & 0,000 & 0 & 0,000 & 9 & 1,215 \\
\hline$L A X A G U E$ & 1 & 9 & 1,402 & 3 & 0,094 & 0 & 0,000 \\
\hline$L A Y$ & 1 & 2 & 0,105 & 9 & 0,239 & 15 & 1,715 \\
\hline LECUONA & 2 & 5 & 1,025 & 1 & 0,017 & 0 & 0,000 \\
\hline$L E M B E Y E$ & 2 & 10 & 0,345 & 11 & 0,820 & 1 & 0,014 \\
\hline LHEZ & 2 & 0 & 0,000 & 0 & 0,000 & 8 & 1,121 \\
\hline$L O N C A$ & 2 & 1 & 0,010 & 0 & 0,000 & 5 & 1,188 \\
\hline$\angle O N C A N$ & 1 & 0 & 0,000 & 2 & 0,017 & 10 & 1,539 \\
\hline LOPEZ & 1 & 12 & 1,161 & 12 & 1,187 & 9 & 0,459 \\
\hline $\operatorname{LOSTE}$ & 2 & 1 & 0,094 & 7 & 0,700 & 6 & 0,459 \\
\hline LOUS TALET & 1 & 8 & 0,241 & 20 & 1,767 & 4 & 0,324 \\
\hline LOUSTALOT & 1 & 10 & 0,481 & 12 & 1,161 & 2 & 0,068 \\
\hline LOUSTAU & 1 & 9 & 0,920 & 20 & 4,584 & 7 & 0,297 \\
\hline LOUSTAUNAU & 1 & 5 & 0,178 & 15 & 2,126 & 1 & 0,014 \\
\hline$L U C U$ & 2 & 6 & 0,764 & 0 & 0,000 & 0 & 0,000 \\
\hline$L U R O$ & 1 & 10 & 1,161 & 1 & 0,102 & 2 & 0,162 \\
\hline MAGENDIE & 2 & 0 & 0,000 & 9 & 0,837 & 2 & 0,027 \\
\hline MAILHARRO & 2 & 5 & 0,743 & 2 & 0,034 & 0 & 0,000 \\
\hline MAILHES & 2 & 0 & 0,000 & 0 & 0,000 & 9 & 0,959 \\
\hline MAIS ONNAVE & 1 & 5 & 0,314 & 9 & 1,152 & 1 & 0,068 \\
\hline MAITIA & 2 & 2 & 1,130 & 0 & 0,000 & 0 & 0,000 \\
\hline MAJESTE & 2 & 1 & 0,031 & 4 & 0,657 & 5 & 0,311 \\
\hline$M A N S E$ & 2 & 0 & 0,000 & 0 & 0,000 & 5 & 1,094 \\
\hline MARMOUGET & 2 & 1 & 0,010 & 1 & 0,009 & 8 & 1,391 \\
\hline$M A R Q U E$ & 1 & 0 & 0,000 & 12 & 0,521 & 11 & 1,877 \\
\hline MARTICORENA & 2 & 8 & 0,826 & 0 & 0,000 & 0 & 0,000 \\
\hline MARTIN & 2 & 6 & 0,450 & 17 & 0,589 & 14 & 1,161 \\
\hline
\end{tabular}




\begin{tabular}{|c|c|c|c|c|c|c|c|}
\hline MARTINEZ & 2 & 9 & 0,847 & 9 & 0,598 & 12 & 0,324 \\
\hline MAUMUS & 2 & 0 & 0,000 & 1 & 0,017 & 12 & 1,310 \\
\hline MAUPOME & 2 & 0 & 0,000 & 0 & 0,000 & 6 & 1,121 \\
\hline MAYSONNAVE & 1 & 2 & 0,094 & 14 & 1,263 & 1 & 0,014 \\
\hline MENDIBOURE & 1 & 10 & 1,935 & 1 & 0,034 & 0 & 0,000 \\
\hline MENDIBURU & 1 & 10 & 1,224 & 0 & 0,000 & 0 & 0,000 \\
\hline MENDIONDO & 2 & 7 & 1,098 & 2 & 0,154 & 0 & 0,000 \\
\hline$M E N D Y$ & 1 & 11 & 1,151 & 0 & 0,000 & 0 & 0,000 \\
\hline MENGELLE & 2 & 1 & 0,010 & 5 & 0,120 & 11 & 0,932 \\
\hline MENVIELLE & 1 & 1 & 0,084 & 3 & 0,111 & 16 & 2,066 \\
\hline MICHELENA & 1 & 7 & 1,506 & 1 & 0,017 & 0 & 0,000 \\
\hline$M I D A N$ & 2 & 0 & 0,000 & 0 & 0,000 & 3 & 0,945 \\
\hline MINVIELLE & 1 & 7 & 0,429 & 21 & 3,859 & 3 & 0,189 \\
\hline$M I Q U E U$ & 2 & 0 & 0,000 & 8 & 0,376 & 8 & 1,121 \\
\hline MIRAMON & 2 & 3 & 0,073 & 14 & 1,042 & 0 & 0,000 \\
\hline MIRANDE & 2 & 8 & 0,743 & 13 & 1,084 & 3 & 0,041 \\
\hline MIRASSOU & 2 & 1 & 0,063 & 14 & 1,024 & 0 & 0,000 \\
\hline MIREMONT & 2 & 7 & 0,764 & 3 & 0,051 & 0 & 0,000 \\
\hline MONLEZUN & 2 & 1 & 0,010 & 2 & 0,017 & 10 & 1,188 \\
\hline MORERE & 2 & 0 & 0,000 & 1 & 0,034 & 6 & 1,242 \\
\hline MOULIA & 2 & 3 & 0,126 & 10 & 0,691 & 1 & 0,041 \\
\hline MOUNIC & 2 & 0 & 0,000 & 2 & 0,017 & 11 & 0,959 \\
\hline MOURA & 2 & 2 & 0,021 & 14 & 0,683 & 5 & 0,230 \\
\hline MOUREU & 2 & 4 & 0,126 & 12 & 0,683 & 4 & 0,284 \\
\hline MOUS QUES & 2 & 0 & 0,000 & 9 & 0,896 & 3 & 0,365 \\
\hline NOBLIA & 2 & 7 & 0,711 & 0 & 0,000 & 0 & 0,000 \\
\hline NOGUE & 1 & 0 & 0,000 & 3 & 0,034 & 7 & 1,458 \\
\hline NOGUES & 1 & 8 & 0,460 & 8 & 0,162 & 19 & 4,226 \\
\hline NOILHAN & 2 & 0 & 0,000 & 0 & 0,000 & 5 & 1,080 \\
\hline OLAIZOLA & 1 & 6 & 1,297 & 0 & 0,000 & 0 & 0,000 \\
\hline OLHAGARAY & 1 & 6 & 1,287 & 0 & 0,000 & 0 & 0,000 \\
\hline OLHARAN & 2 & 8 & 0,795 & 0 & 0,000 & 0 & 0,000 \\
\hline OS PITAL & 1 & 10 & 2,468 & 1 & 0,009 & 0 & 0,000 \\
\hline OUSSET & 2 & 0 & 0,000 & 1 & 0,009 & 4 & 0,959 \\
\hline$O X O B Y$ & 2 & 7 & 1,088 & 1 & 0,017 & 0 & 0,000 \\
\hline OYHARCABAL & 2 & 7 & 1,035 & 0 & 0,000 & 0 & 0,000 \\
\hline OYHENART & 1 & 7 & 1,171 & 0 & 0,000 & 0 & 0,000 \\
\hline PAILHE & 1 & 0 & 0,000 & 4 & 0,154 & 9 & 1,796 \\
\hline PAMBRUN & 1 & 1 & 0,010 & 3 & 0,043 & 8 & 1,404 \\
\hline PARIS & 2 & 9 & 1,109 & 11 & 0,487 & 4 & 0,095 \\
\hline PEHAU & 2 & 0 & 0,000 & 10 & 0,691 & 0 & 0,000 \\
\hline PENE & 1 & 1 & 0,010 & 9 & 0,359 & 15 & 1,998 \\
\hline PERE & 1 & 4 & 0,073 & 19 & 1,878 & 24 & 3,632 \\
\hline PERES & 1 & 5 & 0,115 & 6 & 0,162 & 18 & 2,349 \\
\hline PEREZ & 2 & 12 & 1,015 & 10 & 0,879 & 16 & 0,878 \\
\hline PETEILH & 2 & 0 & 0,000 & 0 & 0,000 & 3 & 0,959 \\
\hline PETRIS SANS & 1 & 12 & 1,945 & 2 & 0,102 & 0 & 0,000 \\
\hline PEYRAN & 1 & 2 & 0,094 & 10 & 1,187 & 1 & 0,014 \\
\hline PEYRE & 1 & 3 & 0,084 & 19 & 1,733 & 8 & 0,297 \\
\hline PEYROU & 2 & 1 & 0,052 & 11 & 0,743 & 6 & 0,608 \\
\hline POCHELU & 1 & 9 & 1,517 & 2 & 0,043 & 0 & 0,000 \\
\hline POEY & 1 & 3 & 0,084 & 15 & 1,229 & 0 & 0,000 \\
\hline POMES & 1 & 0 & 0,000 & 6 & 0,222 & 12 & 1,998 \\
\hline PORTE & 2 & 2 & 0,042 & 8 & 0,862 & 10 & 0,986 \\
\hline PORTERIE & 2 & 1 & 0,010 & 1 & 0,009 & 14 & 0,891 \\
\hline POUBLAN & 1 & 1 & 0,031 & 15 & 1,511 & 2 & 0,081 \\
\hline
\end{tabular}




\begin{tabular}{ll|cc|cc|cc|} 
TOUJAS & 1 & 1 & 0,031 & 0 & 0,000 & 12 & 2,552 \\
TOULET & 1 & 7 & 0,554 & 13 & 1,144 & 1 & 0,027 \\
TOUYA & 1 & 7 & 0,251 & 15 & 1,187 & 8 & 0,392 \\
TREY & 2 & 1 & 0,010 & 2 & 0,222 & 11 & 1,337 \\
TUJAGUE & 2 & 0 & 0,000 & 0 & 0,000 & 7 & 1,094 \\
TURON & 1 & 1 & 0,010 & 17 & 1,998 & 8 & 0,540 \\
UHALDE & 2 & 8 & 1,004 & 0 & 0,000 & 0 & 0,000 \\
UHART & 1 & 11 & 1,653 & 2 & 0,111 & 0 & 0,000 \\
URRUTIA & 1 & 10 & 1,663 & 0 & 0,000 & 0 & 0,000 \\
URRUTY & 1 & 9 & 3,284 & 3 & 0,102 & 0 & 0,000 \\
VEDERE & 1 & 0 & 0,000 & 2 & 0,017 & 10 & 1,688 \\
VERDIER & 1 & 3 & 0,031 & 3 & 0,068 & 16 & 3,294 \\
VERDOUX & 1 & 0 & 0,000 & 1 & 0,026 & 8 & 1,836 \\
VERGEZ & 1 & 11 & 1,213 & 13 & 1,451 & 15 & 2,633 \\
VIAU & 1 & 0 & 0,000 & 4 & 0,154 & 8 & 2,498 \\
VIDAILHET & 2 & 0 & 0,000 & 1 & 0,009 & 8 & 0,891 \\
VIDAL & 2 & 4 & 0,073 & 8 & 0,094 & 13 & 0,999 \\
VIGNAU & 1 & 9 & 0,460 & 22 & 2,996 & 7 & 0,729 \\
VIGNEAU & 1 & 7 & 0,345 & 15 & 1,093 & 9 & 0,284 \\
VIGNES & 2 & 6 & 0,251 & 9 & 0,213 & 15 & 1,364 \\
ZAMORA & 2 & 4 & 0,910 & 0 & 0,000 & 2 & 0,027 \\
ZOZAYA & 2 & 6 & 0,868 & 0 & 0,000 & 0 & 0,000
\end{tabular}


Annexe 2 : Distribution cantonale des fréquences de variantes orthographiques de quelques patronymes.
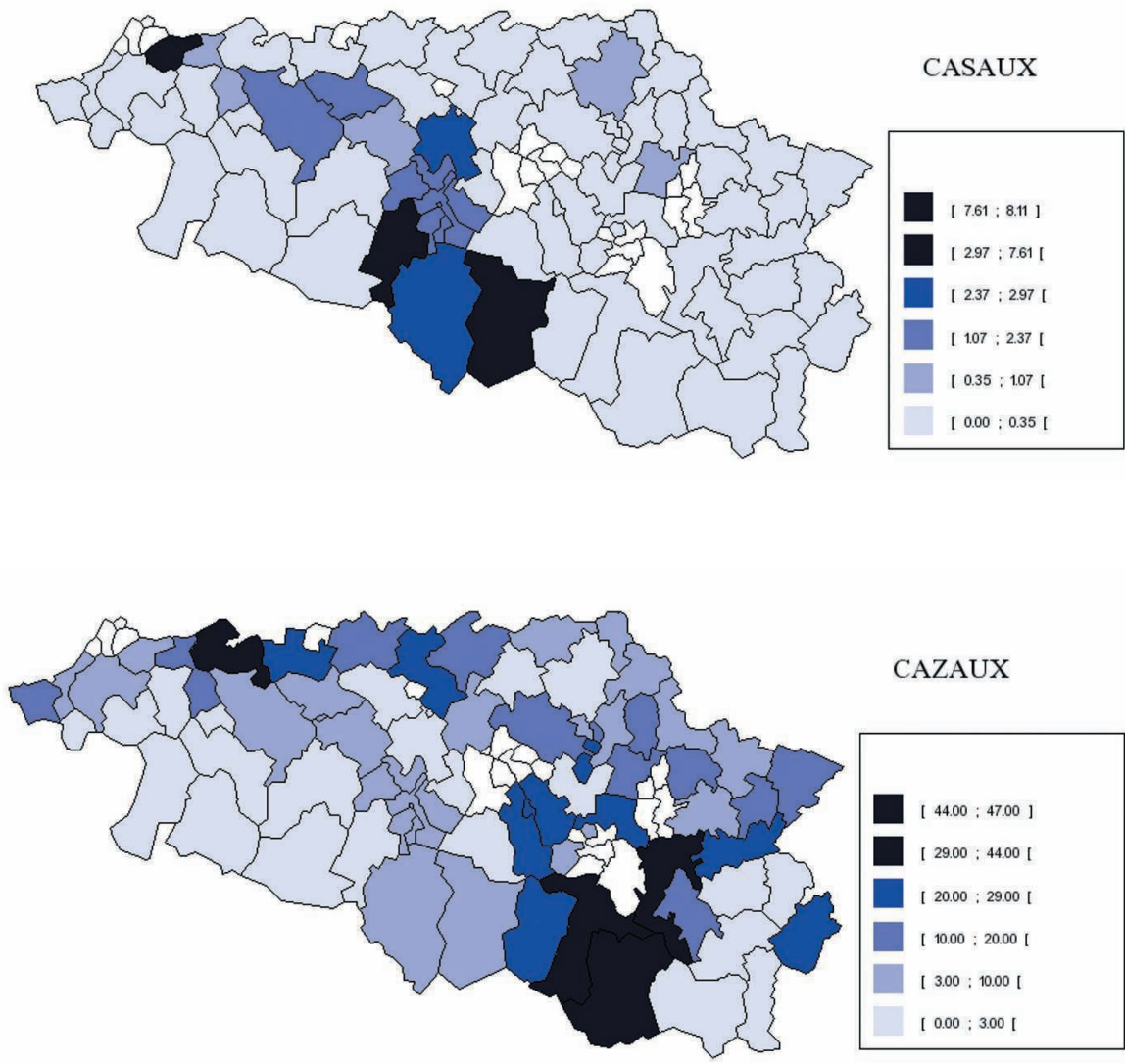

CAZAUX
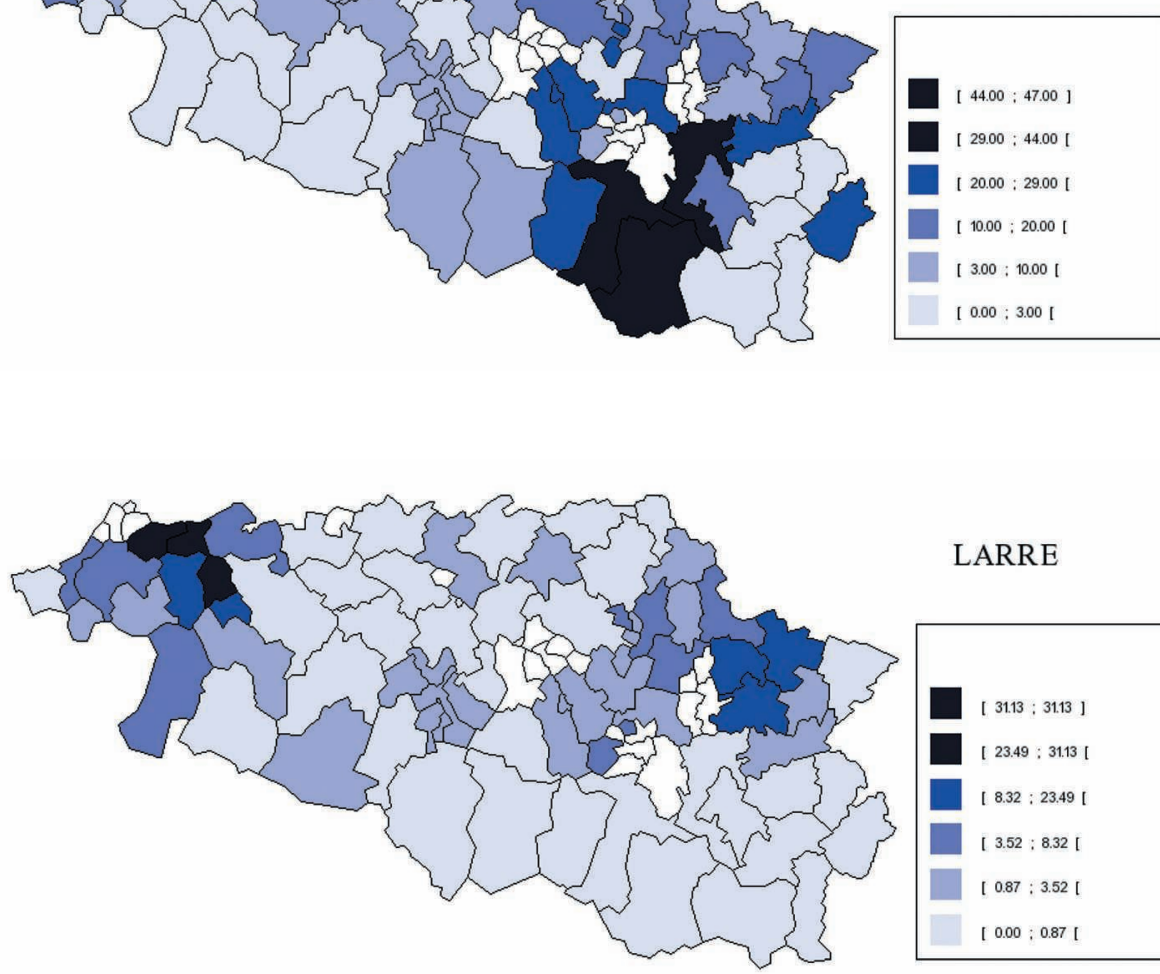

\section{LARRE}

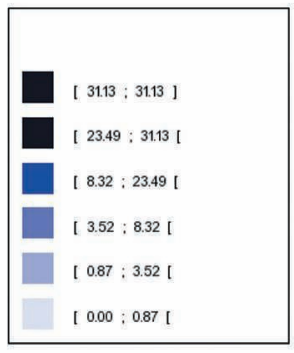



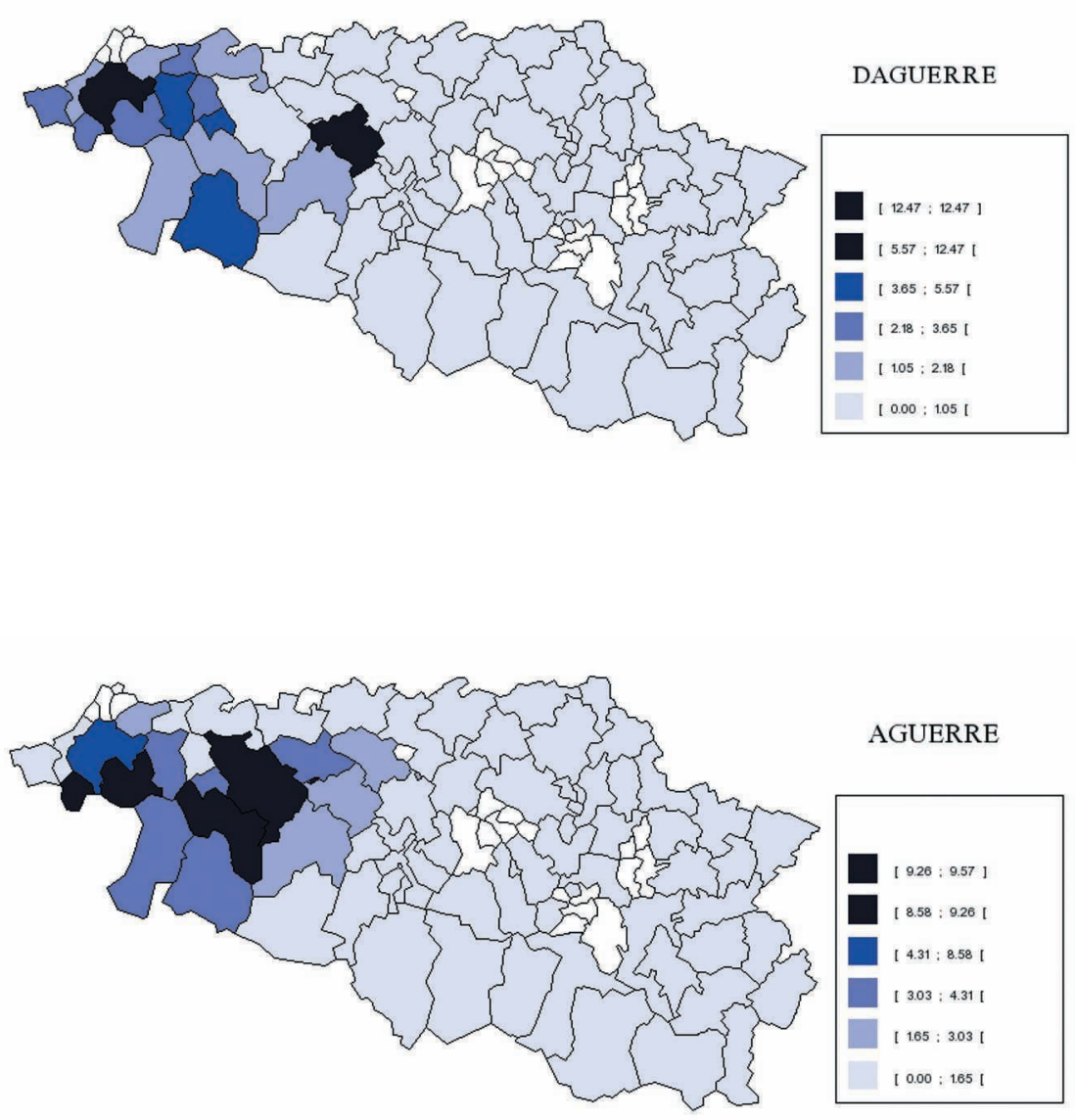

AGUERRE
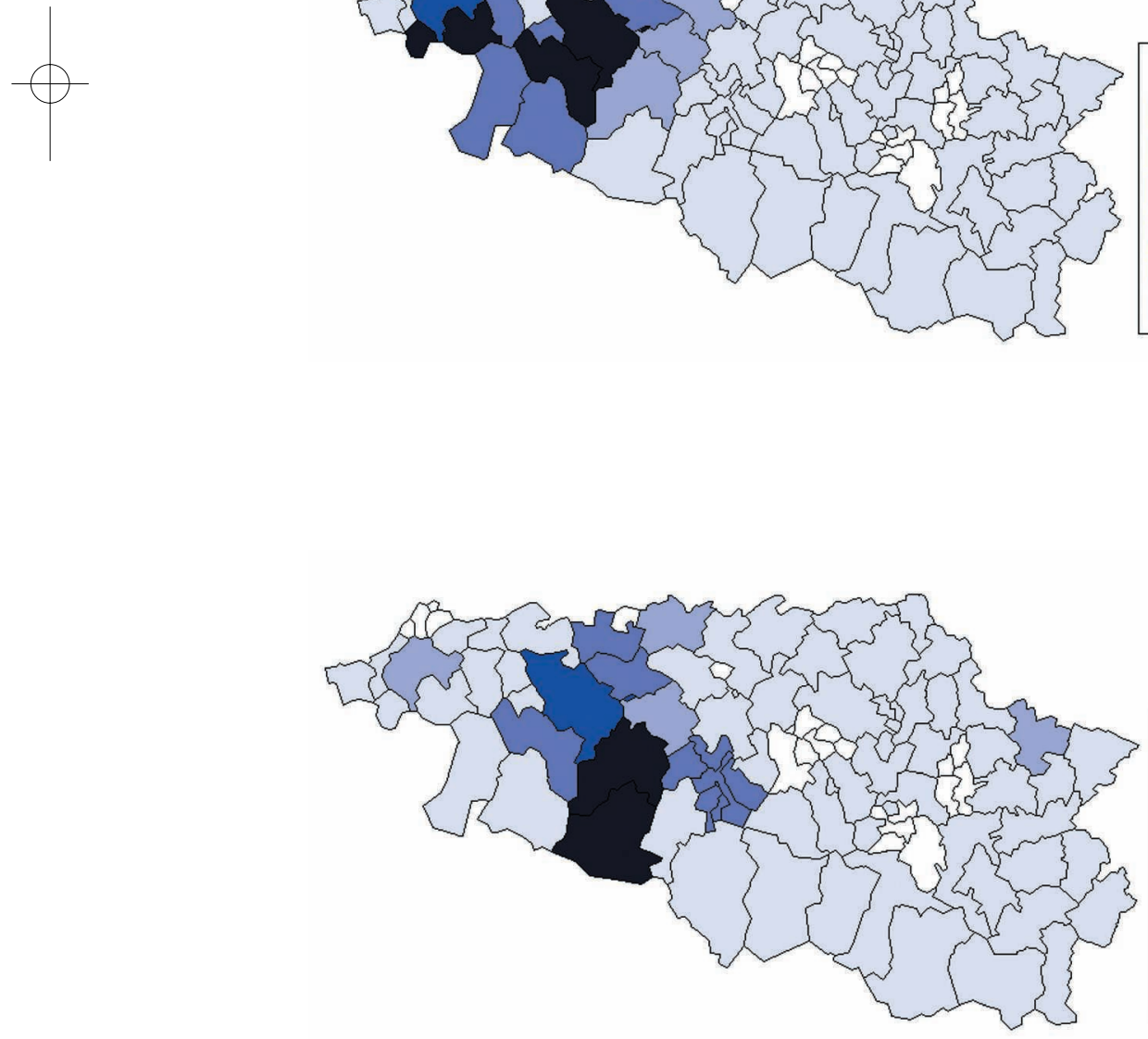

AGUER

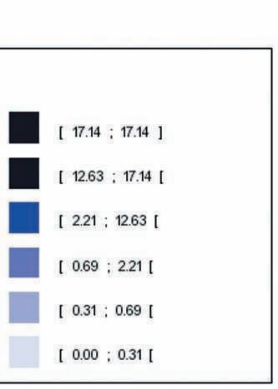



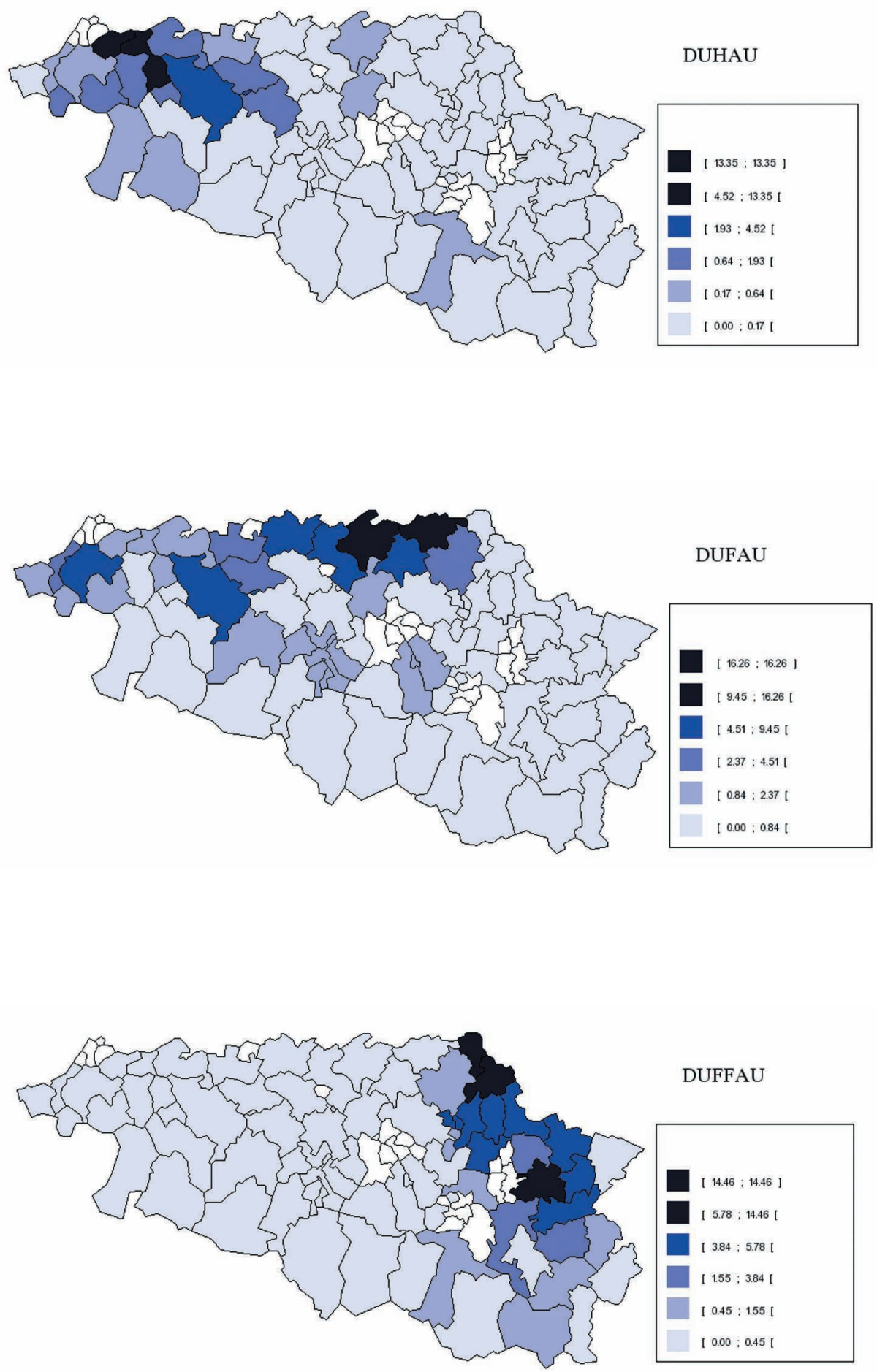

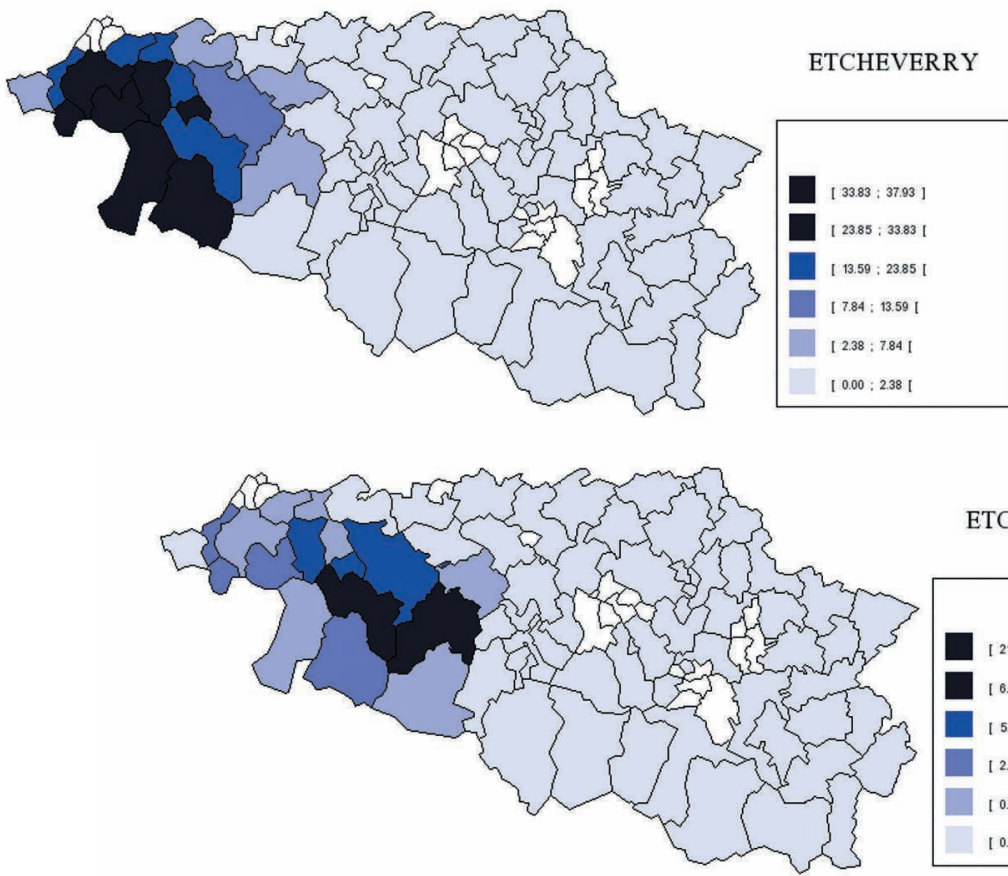

ETCHEBERRY
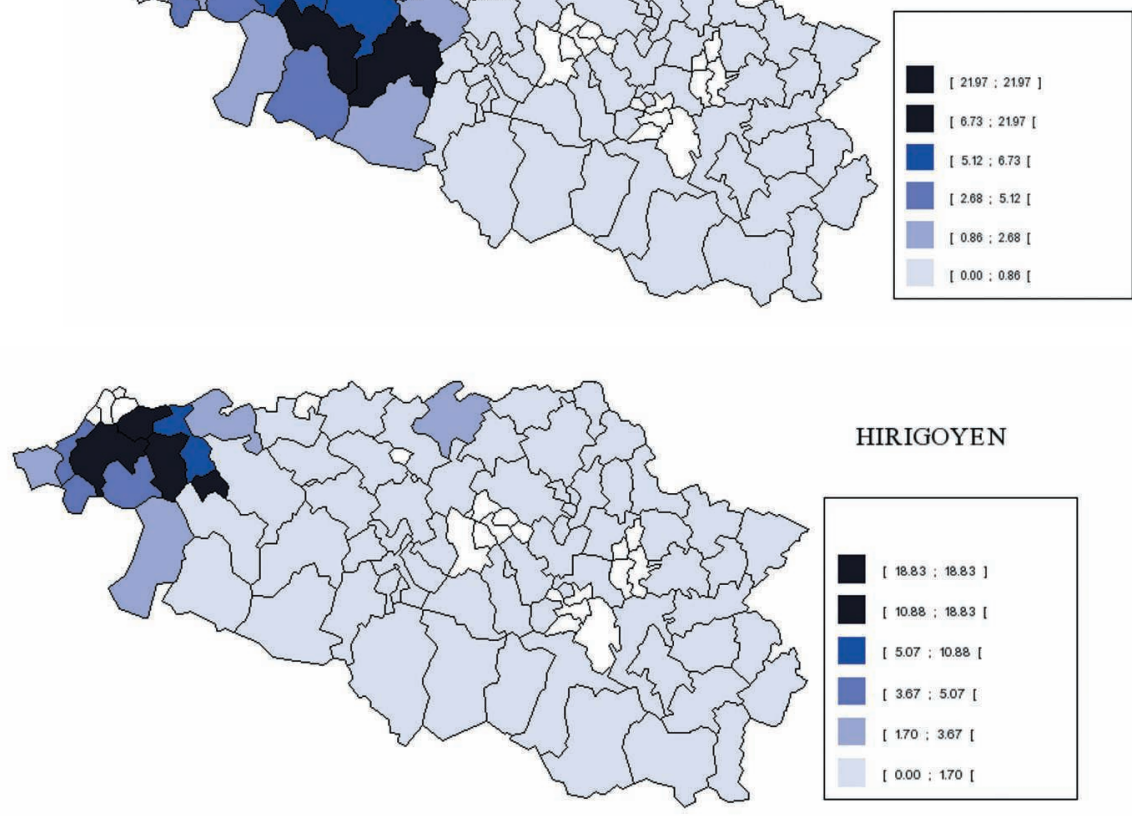

HIRIGOYEN
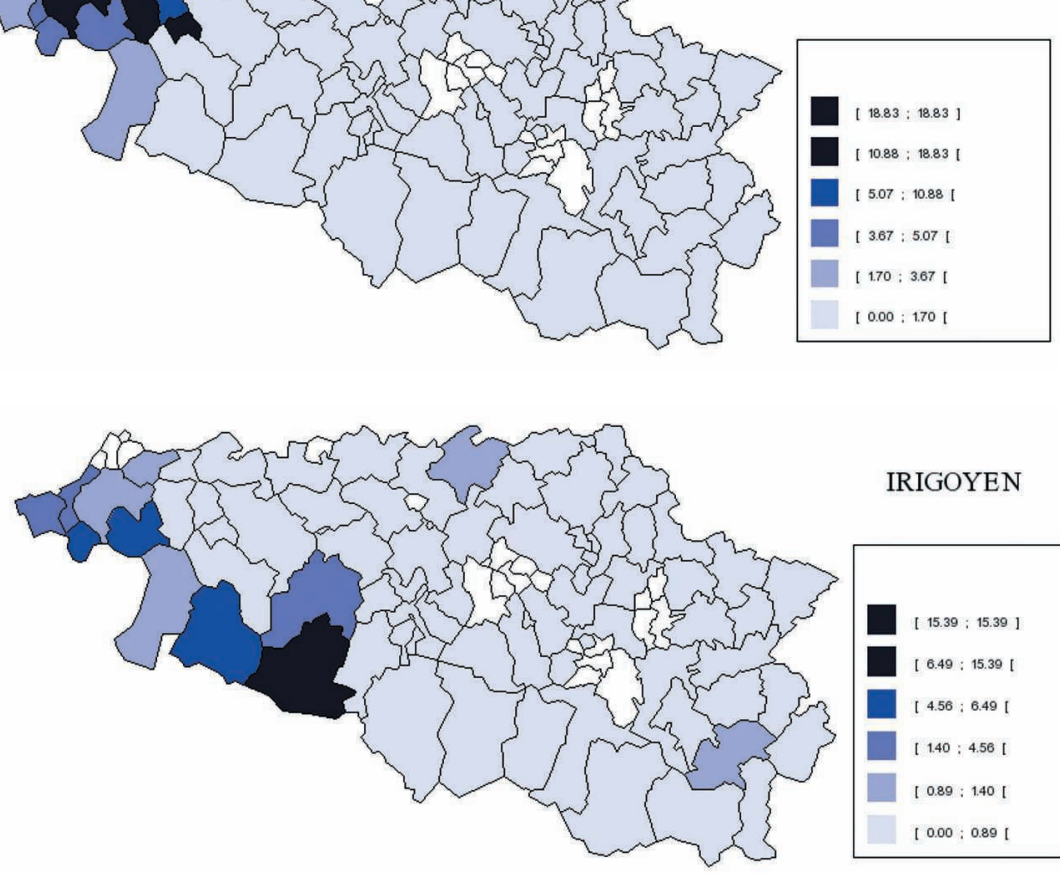

IRIGOYEN

[ $15.39: 15.39]$

[ $6.49: 15.39$

[ $4.56: 6.49$ ]

[ $1.40: 4.56$ ]

[ $0.89: 1.40$ ]

[ $0.00: 0.89$ ] 

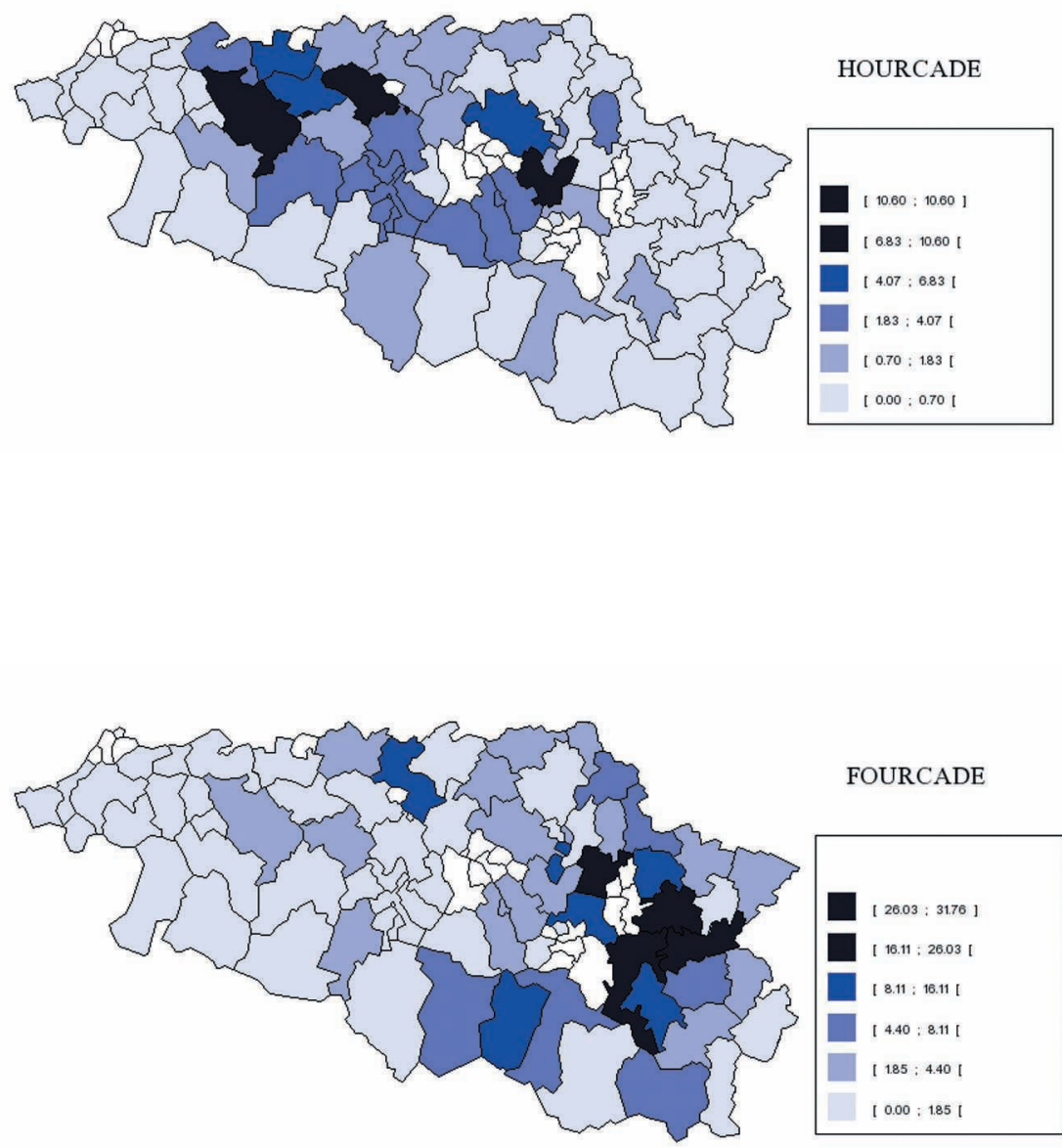